TRANSACTIONS OF THE

AMERICAN MATHEMATICAL SOCIETY

Volume 364, Number 7, July 2012, Pages 3555-3584

S 0002-9947(2012)05402-X

Article electronically published on March 8, 2012

\title{
MAXIMAL FUNCTIONS AND THE ADDITIVITY OF VARIOUS FAMILIES OF NULL SETS
}

\author{
JURIS STEPRĀNS
}

\begin{abstract}
It is shown to be consistent with set theory that every set of reals of size $\aleph_{1}$ is null yet there are $\aleph_{1}$ planes in Euclidean 3-space whose union is not null. Similar results are obtained for circles in the plane as well as other geometric objects. The proof relies on results from harmonic analysis about the boundedness of certain maximal operators and a measure-theoretic pigeonhole principle.
\end{abstract}

\section{INTRODUCTION}

Davies has shown [2] that any measurable subset of the plane can be covered by a family of lines whose union has the same measure as the set itself. A question in this same spirit, due to Peter Komjáth, asked [6] whether the assertion that every set of reals of size $\aleph_{1}$ is Lebesgue null implies that the union of any $\aleph_{1}$ lines in the plane is also Lebesgue null. This was a prime motivation behind the paper [11] which showed that, for any $\gamma<1$, it is consistent that there is a set of reals of size $\aleph_{1}$ which is not null with respect to Hausdorff $\gamma$-measure but all sets of size $\aleph_{1}$ are null with respect to Lebesgue measure. It has been remarked that a Besicovitch duality argument can be used to obtain $\aleph_{1}$ lines in the plane whose union is not Lebesgue null from a set of reals of size $\aleph_{1}$ which is not null with respect to Hausdorff $\gamma$-measure for $\gamma>1 / 2$, thus solving Komjáth's question.

The argument uses the map $L$ from $\mathbb{R}^{2}$ to lines in the plane defined by setting $L(a, b)$ to be the line with slope $a$ and $y$-intercept $b$. For any set $S \subseteq \mathbb{R}^{2}$ and any angle $\theta$, elementary arguments — such as those in $\S 12.1$ of [5] or $\S 7.3$ of [4] - show that the projection of $S$ onto a line forming angle $\theta$ with the $x$-axis is a linear image of the intersection of $L(S)=\bigcup_{(a, b) \in S} L(a, b)$ with the vertical line intersecting the $x$-axis at $\tan (\theta)$. If $L(S)$ is a Lebesgue null set, then there is a null $G_{\delta}$ set $A \supseteq L(S)$ and, furthermore, the set $A^{*}=\{(a, b) \mid L(a, b) \subseteq A\}$ is Borel, contains $S$ and $L\left(A^{*}\right) \subseteq A$. Hence for almost all angles $\theta$ the projection of $A^{*}$ onto the line forming angle $\theta$ with the $x$-axis is null. Since $A^{*}$ is Borel, the Projection Theorem - Theorem 6.1 of [5] or Theorem 6.8 of 4 - implies that the Hausdorff dimension of $A^{*}$, and hence $A$, is less than 1 . Therefore the model of 11 with $1 / 2<\gamma<1$ will solve Komjáth's problem since Theorem 5.8 of [4] implies that the

Received by the editors January 12, 2005 and, in revised form, September 8, 2006, August 4, 2009, May 17, 2010 and June 16, 2010.

2010 Mathematics Subject Classification. Primary 03E17, 42B25.

Key words and phrases. Maximal operator, Besicovitch set, Kakeya set, cardinal invariant, proper forcing.

Research for this paper was partially supported by NSERC of Canada.

(C)2012 American Mathematical Society Reverts to public domain 28 years from publication 
square of a linear set of positive $\gamma$-Hausdorff measure will be a subset of the plane of positive $2 \gamma$-Hausdorff measure. Also observe that complex inversion - in other words, the map sending $z$ to $1 / z$ in the complex plane - sends lines to circles and preserves null sets. Therefore there are also $\aleph_{1}$ circles whose union is not null in this same model.

However, the geometric nature of duality arguments limits the generality one can expect to obtain from them. After all, Komjáth's question can be set in a much broader context by using the framework of small cardinals. For any family $\mathcal{B}$ of compact subsets of a Polish measure space $(X, \mu)$ define the cardinal $\operatorname{add}(\mathcal{B})$ to be the least cardinal of a subfamily $\mathcal{F} \subseteq \mathcal{B}$ such that $\bigcup \mathcal{F}$ is not $\mu$-null. So, letting $\mathcal{S}$ be the points in $\mathbb{R}$ and $\mathcal{P}^{n}$ the hyperplanes in $\mathbb{R}^{n}$, Komjáth's question becomes whether or not $\operatorname{add}(\mathcal{S})=\operatorname{add}\left(\mathcal{P}^{2}\right)$. (Note that $\operatorname{add}(\mathcal{S})$ is the well-known cardinal usually denoted by non $(\mathcal{N} u l l)$.) Letting $\mathcal{C}^{n}$ be the surfaces of spheres in $\mathbb{R}^{n}$ one can ask about the relationships between $\operatorname{add}\left(\mathcal{C}^{n}\right)$ and $\operatorname{add}\left(\mathcal{P}^{k}\right)$ as well as other similarly defined invariants. One cannot hope to apply duality arguments to classes $\mathcal{B}$ which consist of much more than planes or spheres or simple geometric transformations of these objects. For example, let $f \subseteq \mathbb{R}^{n}$ be a smooth curve and let $\mathcal{B}$ consist of all isometric images of $f$. However, there are results in harmonic analysis which allow a solution to the generalized Komjáth problem considerably broader than that provided by 11 .

These results are connected to the problem of reconstructing a measurable function from averages over small sets. The prototypical example here is the Lebesgue Density Theorem which establishes that the value of a measurable function at almost any point $x \in \mathbb{R}^{k}$ can be approximated by taking averages over small balls centred at $x$. If the goal is to approximate the value of the function by averaging over even smaller sets, then, in many cases, this is also possible. For example, Stein [12] showed how to do this for the surfaces of spheres in $\mathbb{R}^{k}$ for $k \geq 3$ and, later, Bourgain [1] did the same for $k=2$. Similar results due to Falconer [3] and Marstrand [7 exist for hyperplanes in $\mathbb{R}^{k}$ for $k \geq 3$ as well. These results, as well as many similar ones, all follow from the boundedness of certain maximal operators associated with the families in question and, in all cases, one gets as a corollary that any set which contains many of the small sets - namely those over which the average is to be calculated - has positive Lebesgue measure.

The main result which will be proved, Theorem 2.1. has as a consequence the consistency of $\operatorname{add}(\mathcal{B})=\aleph_{1} \neq \operatorname{add}(\mathcal{S})$ so long as the boundedness of the maximal operator appropriate for the family $\mathcal{B}$ can be established. The major step in proving Theorem 2.1 will be to use the norms on possibilities technology developed in [10] to construct an $\omega^{\omega}$-bounding, proper partial order which forces the ground model reals to be a null set. In $\$ 2$ the forcing partial order is defined and its main properties are established. The partial order $\mathbb{P}$ will consist of trees of approximations to a cover of the ground model by a null $G_{\delta}$. The strategy for constructing $\mathbb{P}$ will require choosing a very quickly growing sequence of integers $\left\{M_{n}\right\}_{n=0}^{\infty}$ and letting $\mathcal{X}_{n}$ consist of all sets $X \subseteq M_{n}$ having size less than $M_{n} / 2^{n}$. These will be used to code open sets of measure less than $2^{-n}$. For each $n$ an $\mathbb{N}$-valued norm $\nu$ will then be defined on $\mathcal{P}\left(\mathcal{X}_{n}\right)$ which will measure the size of subsets of $\mathcal{X}_{n}$. The trees belonging to $\mathbb{P}$ are then defined to be subtrees of $\prod_{n}^{<\infty} \mathcal{X}_{n}$ such that for each $t \in T$ and each integer $k$ there is some $s \supseteq t$ such that $\nu\left(\Sigma_{T}(s)\right) \geq k$, where $\Sigma_{T}(s)$ is the set of successors of $s$. It will be easy to see that $\mathbb{P}$ forces the ground model 
reals to become a null set, but to see why, for example, the union of the ground model circles is not Lebesgue null after forcing with such a partial order requires looking at the construction of the norm $\nu$ using the boundedness of the appropriate maximal operator.

This construction is completed in $\$ 4$ by proving a measure-theoretic pigeonhole principle which may be of interest in its own right as well. In the simplest 1dimensional case, the principle says that a measurable mapping of a product of probability spaces either maps most of a vertical section to a small set, or, it maps most of the graph of a function to a small set. The proof provided in $₫ 4$ works only for finite probability spaces unless some additional axioms are assumed, but this is enough for the intended applications. The connection between this pigeonhole principle and the norm of $₫ 2$ is established in $\$ 3$ by introducing an intermediary norm which bridges the gap between maximal functions and pigeonhole principles.

The applications of the theorem, as well as the results from harmonic analysis upon which they are based, are stated in $\$ 5$. Some final remarks and questions are also recorded in $\$ 5$.

\section{DEFINING THE PARTIAL ORDER}

The study of the additivity properties of the families mentioned in the introduction will rely a great deal on the fact that these families are not only easily definable, but that they also have well-behaved parameterizations by measure spaces. These parameterizations by measure spaces are crucial in that they enable arguments to use concepts such as many circles or most lines. Definition 2.1 describes the sort of parametrized families which will be studied.

Definition 2.1. Let $(P, \sigma)$ be an atomless Polish probability space and let $\operatorname{Compact}\left(\mathbb{R}^{n}\right)$ be the space of compact subsets of $\mathbb{R}^{n}$ under the Hausdorff metric. Let $\lambda$ be Lebesgue measure on $\mathbb{R}^{k}$ and $\mu$ be a regular measure on $\mathbb{R}^{k}$ such that the set

$$
\left\{C \subseteq \operatorname{Compact}\left(\mathbb{R}^{k}\right) \mid \mu(C)>r\right\}
$$

is Borel in Compact $\left(\mathbb{R}^{n}\right)$. A mapping $\Lambda$ defined on $P$ will be said to be $\mu$-appropriate if:

(1) $\Lambda: P \rightarrow \operatorname{Compact}\left(\mathbb{R}^{n}\right)$ is continuous.

(2) $1 \leq \inf _{p \in P} \mu(\Lambda(p)) \leq \sup _{p \in P} \mu(\Lambda(p))<\infty$.

(3) For every $\theta>0$ and $\delta>0$ if $J(\theta, \delta)$ is defined by

$$
\begin{gathered}
J(\theta, \delta)=\inf \left\{\lambda(A) \mid A \subseteq \mathbb{R}^{n} \text { is open and } \sigma(\{p \in P \mid \mu(\Lambda(p) \cap A)>\theta\})>\delta\right\}, \\
\text { then } J(\theta, \delta)>0 \text { and } \lim _{\delta \rightarrow 0} J(\theta, \delta)=0 \text { for all } \theta .
\end{gathered}
$$

In all the cases to be considered here the measure $\mu$ will be Hausdorff $k$-measure for some integer $k$. Observe that all Hausdorff $k$-measures $\mu$ satisfy the property that the set

$$
A=\left\{(C, r) \mid C \subseteq \mathbb{R}^{k} \text { is compact and } \mu(C)>r\right\}
$$

is Borel in $\operatorname{Compact}\left(\mathbb{R}^{n}\right) \times \mathbb{R}$. To see this, observe that $(C, r) \notin A$ if and only if for each integer $m$ there are $\left\{q_{i}\right\}_{i=1}^{k} \subseteq \mathbb{R}^{n}$ with rational coordinates and rational $\left\{t_{i}\right\}_{i=1}^{k}$ such that $0, t_{i}<1 / m$ for all $i$ and $\sum_{i=1}^{k} t_{i}^{s} \leq r$ and

$$
C \subseteq \bigcup_{i=1}^{k} B_{t_{i}}\left(q_{i}\right),
$$


where $B_{t_{i}}\left(q_{i}\right)$ represents the open ball of diameter $t_{i}$ and centre $q_{i}$. Compactness yields $\epsilon>0$ such that the open ball around $C$ in the Hausdorff metric with radius $\epsilon$ is contained in $\bigcup_{i=1}^{k} B_{t_{i}}\left(q_{i}\right)$. In other words, $A$ is $F_{\sigma}$.

An example might be useful at this point.

Example 2.1. Let $(P, \sigma)=([1,2], \lambda)$ and let $\Lambda: P \rightarrow \operatorname{Compact}\left(\mathbb{R}^{k}\right)$ be $\operatorname{defined}$ by letting $\Lambda(t)$ be the surface of the $k$-sphere of radius $t$ with centre at the origin. In this case $\Lambda$ is $\lambda^{2}$-appropriate where $\lambda^{2}$ is Hausdorff 2-measure, noting that this restricts to surface measure on each sphere. The fact that conditions 1 and 2 are satisfied is clear. That condition 3 holds can be seen by applying Fubini's Theorem in spherical coordinates.

The following two examples illustrating Definition 2.1, in spite of their similarity, will provide boundaries for the application of the methods to be developed; those methods will be applicable to Example 2.2, but not to Example 2.3.

Example 2.2. Let $(P, \sigma)=((0,1], \lambda)$. Let $\Lambda: P \rightarrow \operatorname{Compact}\left(\mathbb{R}^{3}\right)$ be defined by letting $\Lambda(t)$ be the disc of radius 1 with centre at $(t, 0,0)$ and perpendicular to the $t$-axis. The fact that the mapping $\Lambda$ is $\lambda^{2}$-appropriate follows from an easy application of Fubini's Theorem.

Example 2.3. Let $(P, \sigma)=((0,1], \lambda)$ and let $\Lambda: P \rightarrow \operatorname{Compact}\left(\mathbb{R}^{2}\right)$ be defined by letting $\Lambda(t)$ be the unit line segment with centre at $(t, 0)$ and perpendicular to the $t$-axis. Once again, Fubini's Theorem shows that the mapping $\Lambda$ is $\lambda^{1}$-appropriate.

The following definition is needed to be able to apply results in the harmonic analysis literature to the context of Definition 2.1

Definition 2.2. Let $(G, \cdot)$ be a locally compact group and let $\lambda_{G}$ be its Haar measure. Let $*$ be a continuous left action of $G$ on $\mathbb{R}^{k}$ that is measure-preserving; in other words, $\lambda(A)=\lambda(g * A)$ for each measurable $A \subseteq \mathbb{R}^{k}$ and $g \in G$. For any $\mu$-appropriate function $\Lambda: P \rightarrow \operatorname{Compact}\left(\mathbb{R}^{k}\right)$ the maximal operator $M_{\Lambda, G}$ can be defined on measurable functions on $\mathbb{R}^{k}$ by

$$
M_{\Lambda}(f)(g)=\sup _{p \in P} \int_{z \in \Lambda(p)} f(g * z) d \mu .
$$

The operator $M_{\Lambda}$ will be said to be bounded if there are real numbers $q \geq 1, p \geq 1$ and a constant $K$ such that

$$
\left\|M_{\Lambda}(f)\right\|_{p} \leq K\|f\|_{q}
$$

for all $f$ which are the characteristic functions of bounded open sets in $\mathbb{R}^{k}$. The norm \|\|$_{p}$ is calculated with respect to $\lambda_{g}$ of course.

Lemma 2.1. If $\Lambda$ is a $\mu$-appropriate function and $(G, \cdot)$ is a locally compact group and $*$ a continuous left action of $G$ on $\mathbb{R}^{k}$, then for every open set $U \subseteq \mathbb{R}^{n}$ the mapping $\Lambda^{U}: P \times G \rightarrow \mathbb{R}$ defined by $\Lambda^{U}(p, g)=\mu((g * \Lambda(p)) \cap U)$ satisfies the following measurability condition: For every $r \in \mathbb{R}$,

$$
\left\{(p, g) \in P \times G \mid \Lambda^{U}(p, g)>r\right\}
$$

is analytic. 
Proof. Note that the inner regularity of $\mu$ implies that the set (2.4) is equal to the set of all $(p, g)$ such that there exists $C \in \operatorname{Compact}\left(\mathbb{R}^{n}\right)$ such that

(1) $C \subseteq g * \Lambda(p)$,

(2) $C \subseteq U$,

(3) $\mu(C)>r$,

and each of these conditions is described by a Borel set.

In particular, $C \nsubseteq g * \Lambda(p)$ if and only if there is $c \in C$ such that the Euclidean distance from $c$ to $g * \Lambda(p)$ is greater than 0 . Using the continuity of $\Lambda$ and the continuity of the action $*$ it follows that there is an open neighbourhood $V$ of $(C, p, g)$ in the space $\operatorname{Compact}\left(\mathbb{R}^{n}\right) \times P \times G$ such that $C^{\prime} \nsubseteq g^{\prime} * \Lambda\left(p^{\prime}\right)$ for each $\left(C^{\prime}, p^{\prime}, g^{\prime}\right) \in V$. In other words, if

$$
A_{1}=\left\{(C, p, g) \in \operatorname{Compact}\left(\mathbb{R}^{n}\right) \times P \times G \mid C \subseteq g * \Lambda(p)\right\},
$$

then $A_{1}$ is closed.

If $A_{2}=\left\{(C, p, g) \in \operatorname{Compact}\left(\mathbb{R}^{n}\right) \times P \times G \mid C \subseteq U\right\}$, then it is clear that $A_{2}$ is open and if

$$
A_{3}=\left\{(C, p, g) \in \operatorname{Compact}\left(\mathbb{R}^{n}\right) \times P \times G \mid \mu(C)>r\right\},
$$

then $A_{3}$ is Borel by the hypothesis of Definition 2.1 .

Therefore the set (2.4) is equal to

$$
\left\{(p, g) \in P \times G \mid(\exists C \in \operatorname{Compact}(G))(C, p, g) \in A_{1} \cap A_{2} \cap A_{3}\right\}
$$

establishing that the set (2.4) is analytic.

Also note that Lemma 2.1 cannot be strengthened to obtain that the mapping $\Lambda^{U}$ is continuous. For example if $s=0, \lambda$ is the counting measure, $P=[0,1]$ and $\Lambda(x)=\{x, x / 2\}$, then $\lim _{x \rightarrow 0} \lambda(\Lambda(x))=2$ while $\lambda(\Lambda(0))=1$.

Defining the operator $M_{\Lambda}$ only for those functions which are the characteristic functions of bounded open sets may seem somewhat contrived, but this unnatural formulation will be useful when applying the results of $\$ 5$. It is therefore useful to observe that for any $\mu$-appropriate function $\Lambda: P \rightarrow \operatorname{Compact}\left(\mathbb{R}^{k}\right),(G, \cdot)$ a locally compact group and * a continuous, measure-preserving left action of $G$ on $\mathbb{R}^{k}$, the maximal operator $M_{\Lambda}$ can be defined for the characteristic functions of bounded open sets $U \subseteq \mathbb{R}^{k}$ by

$$
M_{\Lambda}(U)(g)=\sup _{p \in P} \Lambda^{U}(p, g) .
$$

In this formulation it is easy to see that $M_{\Lambda}(U)$ is a measurable function. To see this observe that $M_{\Lambda}(U)(g)>r$ if and only if

$$
(\exists p \in P) \Lambda^{U}(p, g)>r
$$

and the set of all $g \in G$ satisfying this condition is analytic, and hence measurable, by Lemma [2.1. It follows that $M_{\Lambda}$ is measurable and inequality (2.3) is then reformulated as

$$
\left\|M_{\Lambda}(U)\right\|_{p} \leq K \lambda(U)^{p / q} .
$$

Since broadening the scope of the definitions of the maximal operators $M_{\Lambda}$ to functions other than characteristic functions of open sets would find no applications in the current work, the implications of the more natural definition will not be pursued here. It should be noted that often the definition of maximal operators involves normalizing the measure $\mu$ by dividing by $\mu(\Lambda(p))$ in equation (2.2). This 
plays no role here because only the boundedness of these operators is of interest and condition (2) of Definition 2.1 is always in force. Also, the supremum is usually taken over all reals but once again, since only boundedness is of interest here, this is not significant.

Proposition 2.1. If $\Lambda: P \rightarrow \operatorname{Compact}\left(\mathbb{R}^{k}\right)$ and $(G, \cdot)$ is a locally compact group and $*$ a continuous left action of $G$ on $\mathbb{R}^{k}$, then for every $\epsilon>0$ and open $A \subseteq[0,1]^{k}$ the following weak type $(p, q)$ inequality holds:

$$
\lambda_{G}\left(\left\{g \in G \mid(\exists x \in P) \Lambda^{A}(x, g) \geq \epsilon\right\}\right) \leq \frac{K \lambda(A)^{p / q}}{\epsilon^{p}}
$$

provided that $\Lambda$ is s-appropriate and $M_{\Lambda}$ satisfies inequality (2.6).

Proof. It follows from inequality (2.6) that

$$
\int_{g \in G} \sup _{x \in P}\left(\Lambda^{A}(x, g)\right)^{p} d \lambda \leq K \lambda(A)^{p / q}
$$

and using that

$$
\lambda\left(\left\{g \in G \mid(\exists x \in P) \Lambda^{A}(x, g) \geq \epsilon\right\}\right) \epsilon^{p} \leq \int_{g \in G} \sup _{x \in P}\left(\Lambda^{A}(x, g)\right)^{p} d \lambda
$$

the result follows.

In the arguments to follow, inequality (2.7) will play a central role and the boundedness of maximal operators will merely be used to conclude that inequality (2.7) holds. Indeed, Marstrand obtains equivalent inequalities in [7] and [8] without resorting to maximal operators. For the remainder of this section, as well as 33 , let $(P, \sigma)$ be an atomless, Polish probability space and $\Lambda: P \rightarrow \mathbb{R}^{k}$ be either $s$-appropriate or a parametrized family of $s$-appropriate functions and let $J:(0,1)^{2} \rightarrow(0,1)$ be as in Definition 2.1. If $\Lambda$ is a parametrized family of $s$ appropriate functions, then let $\left(P_{0}, \sigma_{0}\right)$ and $\left(P_{1}, \sigma_{1}\right)$ be Polish probability spaces as required in Definition 2.1 such that $P=P_{0} \times P_{1}$ and $\sigma=\sigma_{0} \times \sigma_{1}$. Also assume that inequality (2.7) holds for $K, q^{\prime}$ and $q$. For example, this will hold if the operator $M_{\Lambda}$ is bounded.

Notation 2.1. If $A \subseteq X \times Y$ and $x \in X$, then $A\langle x\rangle=\{y \in Y \mid(x, y) \in A\}$.

Definition 2.3. Let $\{\epsilon(j)\}_{j=1}^{\infty}$ be a descending sequence of positive reals such that $\epsilon(1)<1$ and for each $j \in \mathbb{N}$ and any two probability spaces $\left(Q_{0}, \mu_{0}\right)$ and $\left(Q_{1}, \mu_{1}\right)$,

$$
\mu_{0}\left(\left\{x \in Q_{0} \mid \mu_{1}(A\langle x\rangle)>2 \epsilon(j)\right\}\right)>2 j^{q^{\prime}} K^{q^{\prime}} J(1 / j, \epsilon(j))^{q^{\prime} / q}
$$

for all $A \subseteq Q_{0} \times Q_{1}$ such that $\mu_{0} \times \mu_{1}(A) \geq 1 /(j+1)$. Let $J^{*}(j)=J(1 / j, \epsilon(j))$.

Definition 2.4. For $M \in \mathbb{N}$ and $\xi>0$ define $\mathcal{P}_{\xi}(M)$ to be the set of all $X \subseteq M$ such that $|X|<\xi M$. Define a function $\Theta: \mathcal{P}\left(\mathcal{P}_{\xi}(M)\right) \rightarrow \mathcal{P}(\mathbb{N})$ by specifying inductively, simultaneously for all $\mathcal{Y} \subseteq \mathcal{P}_{\xi}(M)$, which integers belong to $\Theta(\mathcal{Y})$.

- For all $\mathcal{Y} \subseteq \mathcal{P}_{\xi}(M), 0 \in \Theta(\mathcal{Y})$ if and only if $\mathcal{Y} \neq \emptyset$.

- For all $\mathcal{Y} \subseteq \mathcal{P}_{\xi}(M), 1 \in \Theta(\mathcal{Y})$ if and only if $\bigcup \mathcal{Y}=M$.

- For all $\mathcal{Y} \subseteq \mathcal{P}_{\xi}(M)$, if $j \geq 1$, then $j+1 \in \Theta(\mathcal{Y})$ if and only if for every function $F$ from $\mathcal{Y}$ to the open subsets of $\mathbb{R}^{k}$ of measure less than $J^{*}(j)$,

$$
\lambda \times \sigma\left(\left\{(x, p) \in[0,1]^{k} \times P \mid j \notin \Theta\left(\langle x, p, F\rangle_{j}\right)\right\}\right) \leq 1 /(j+1),
$$


where $\langle x, t, H\rangle_{n}$ is defined to be $\left\{Y \mid \Lambda^{H(Y)}(t, x) \leq 1 / n\right\}$ for $t \in P, x \in$ $[0,1]^{k}$, any integer $n$ and any partial function $H$ from $\mathcal{P}\left(\mathcal{P}_{\xi}(M)\right)$ to the open subsets of $\mathbb{R}^{k}$. Observe that the set of inequality (2.11) is measurable by Lemma 2.1 .

Define $\nu(\mathcal{Y})=j$ if $j+1$ is the least integer such that $j+1 \notin \Theta(\mathcal{Y})$.

Observe that if $\mathcal{Y}_{1} \subseteq \mathcal{Y}_{2}$, then $\nu\left(\mathcal{Y}_{1}\right) \leq \nu\left(\mathcal{Y}_{2}\right)$ and if $j+1 \in \Theta(\mathcal{Y})$, then $j \in \Theta(\mathcal{Y})$. Now note that since $1 /(j+1)<1$ for each $j \geq 1$ it follows that if $\nu(\mathcal{Y}) \geq j+1$, then, letting $F$ be the function defined by $F(Y)=\emptyset$, it follows that there is some pair $(x, p)$ such that $\nu\left(\langle x, p, F\rangle_{j}\right) \geq j$. Applying this successively yields a subset $\mathcal{Z} \subseteq \mathcal{Y}$ such that $\nu(\mathcal{Z}) \geq 1$ or, in other words, $\bigcup \mathcal{Z}=M$.

At this point it is not even clear whether there is any integer $M$ such that $\nu\left(\mathcal{P}_{1 / 2}(M)\right)>2$. This, and much more, will be resolved in 4 . For the moment, however, the following is stated as a hypothesis to be justified later in Corollary 4.1

$$
(\forall n \in \mathbb{N})(\exists M(n) \in \mathbb{N}) \nu\left(\mathcal{X}_{n}\right)>n,
$$

where $\mathcal{X}_{n}=\mathcal{P}_{2^{-n}}(M(n))$. Let $T=\prod_{n=0}^{\infty} \mathcal{X}_{n}$ and let $T^{<\infty}=\bigcup_{i=0}^{\infty} \prod_{n=0}^{i} \mathcal{X}_{n}$. For $t \in T^{<\infty}$ let $[t]$ denote the open subset of $T$ defined by $[t]=\{c \in T \mid t \subseteq c\}$. Now for any closed $C \subseteq T$ let $C^{<\infty}=\left\{t \in T^{<\infty} \mid[t] \cap C \neq \emptyset\right\}$ and, for $t \in C^{<\infty}$ let $\Sigma_{C}(t)$ be the immediate successors of $t$ in $C^{<\infty}$; in other words,

$$
\Sigma_{C}(t)=\left\{X \in \mathcal{X}_{|t|} \mid(\exists c \in C) t \subseteq c \text { and } c(|t|)=X\right\} .
$$

Let $\mathbb{P}$ consist of all closed subsets of $C \subseteq T$ such that for all $t \in C^{<\infty}$ and for each $j \in \mathbb{N}$ there is some $s \in C^{<\infty}$ such that $t \subseteq s$ and $\nu\left(\Sigma_{C}(s)\right) \geq j$. For an ordinal $\xi$ let $\mathbb{P}_{\xi}$ be the countable support product of $\xi$ copies of $\mathbb{P}$ and let $\leq$ be the coordinate-wise ordering on $\mathbb{P}_{\xi}$. Standard fusion arguments show that $\mathbb{P}_{\xi}$ partially ordered by $\leq$ is proper. Before continuing it will be observed that if $\kappa$ is a regular cardinal, then

$$
1 \Vdash_{\mathbb{P}_{\kappa}} \cdot \operatorname{add}(\mathcal{S})=\operatorname{non}(\mathcal{N} u l l) \geq \kappa "
$$

and, indeed, this follows from the next lemma.

Lemma 2.2. If $V \subseteq W$ are both models of set theory, then

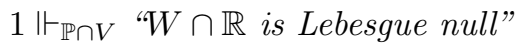

in the model $W$.

Proof. For $A \in \mathcal{X}_{n}$ define

$$
A^{*}=\bigcup_{i \in A}\left[\frac{i}{M(n)}, \frac{i+1}{M(n)}\right]
$$

and let

$$
S_{m}(G)=\bigcup_{n=m+1}^{\infty} G(n)^{*},
$$

where $G$ is a name for the $\mathbb{P}$ generic real. It follows that the Lebesgue measure of $S_{m}(G)$ is no greater than $1 / 2^{m}$ and it suffices to show that given $C \in \mathbb{P} \cap V$, $m \in \mathbb{N}$ and $x \in[0,1]$ in the model $W$ there is some $C^{\prime} \subseteq C$ such that $C^{\prime} \in \mathbb{P} \cap V$ and $C^{\prime} \Vdash_{\mathbb{P} \cap V}$ " $x \in S_{m}(G)$ ". Choose $t \in C^{<\infty}$ such that $\nu\left(\Sigma_{C}(t)\right) \geq 1$ and $|t| \geq m$. Then, since $\bigcup \Sigma_{C}(t)=M(|t|)$ and this is absolute, it is possible to select $X \in \Sigma_{C}(t)$ such that $x \in X^{*}$. Then let $C^{\prime}=C \cap\left[t^{\frown} X\right]$. 
Corollary 2.1. If $\kappa$ is a regular cardinal, then $1 \Vdash_{\mathbb{P}_{\kappa}} " \operatorname{add}(\mathcal{S})=\operatorname{non}(\mathcal{N} u l l) \geq \kappa "$.

Proof. Given $G \subseteq \mathbb{P}_{\kappa}$ generic over $V$ and $X \subseteq \mathbb{R}$ of cardinality less than $\kappa$ in $V[G]$, the properness of $\mathbb{P}_{\kappa}$ guarantees that there is $\xi \in \kappa$ such that $X \in W=V\left[G \cap \mathbb{P}_{\xi}\right]$. Lemma 2.2 now applies.

In the following, note that the continuity of $\Lambda$ ensures that $\Lambda$ is defined in any model of set theory so that $\Lambda(p)$ is always interpreted as a compact set.

Theorem 2.1. For any cardinal $\kappa$

$$
1 \Vdash_{\mathbb{P}_{\kappa}} \text { " } \bigcup_{x \in V \cap \mathbb{R}^{k}} \bigcup_{v \in V \cap P}(\Lambda(v)+x) \text { is not Lebesgue null". }
$$

Proof. The proof will be presented in a series of steps, each adding another level of complexity.

To begin, it will be assumed that $\kappa=1$ and $\Lambda$ is $s$-appropriate. If the theorem is false, then, without any loss of generality, there is $p \in \mathbb{P}$ such that

$$
p \Vdash_{\mathbb{P}} " \bigcup_{x \in V \cap \mathbb{R}^{k}} \bigcup_{v \in V \cap P}(\Lambda(v)+x) \subseteq \bigcup_{i=0}^{\infty} \prod_{j=1}^{k}\left(q_{j, i}, q_{j, i}+r_{i}\right) \text { and } \sum_{i=0}^{\infty} r_{i}^{k}<J(1 / 2,1 / 3) ",
$$

where it may be assumed that $r_{i}$ and $q_{j, i}$ are names for rationals. The cube $\prod_{j=1}^{k}\left(q_{j, i}, q_{j, i}+r_{i}\right)$ will be represented by $B_{i}$.

Now construct by induction on $n$ conditions $p_{n} \in \mathbb{P}$, integers $N_{n}$ and $K_{n} \geq 1$ and $A_{n} \subseteq T^{<\infty}$ for $i \leq n$ such that:

(1) $p_{0} \leq p$ and $p_{n+1} \leq p_{n}$ for each $n$.

(2) $\{[t]\}_{t \in A_{n}}$ is a finite open cover of $p_{n}$ by disjoint non-empty sets.

(3) If $t \in A_{n}$, then $|t|>n$.

(4) $\Sigma_{p_{n}}(t)=\Sigma_{p_{m}}(t)$ for each $m \geq n$ and for each $t \in A_{n}$.

(5) $\nu\left(\Sigma_{p_{n}}(t)\right) \geq K_{n}+1 \geq n$ for each $t \in A_{n}$.

(6) There is an open set $W$ such that $p_{0} \Vdash$ " $\bigcup_{i=0}^{N_{0}} B_{i}=\check{W}$ ".

(7) For each $t \in A_{n}$ and $Y \in \Sigma_{p_{n}}(t)$ there is an open set $W_{t \frown Y}$ such that

$$
p_{n+1} \cap\left[t^{\frown} Y\right] \Vdash \text { " } \bigcup_{i=N_{n}+1}^{N_{n+1}} B_{i}=\check{W}_{t \frown Y} \text {. }
$$

(8) $p_{n} \Vdash " \sum_{i=N_{n}+1}^{\infty} r_{i}^{k}<J^{*}\left(K_{n}\right) "$.

(9) $\sum_{i=0}^{n} \frac{\left|A_{i}\right|}{K_{i}+1}<1 / 2$.

Given that this can be done, define $p_{\omega}=\bigcap_{n=0}^{\infty} p_{n}$ and note that each $A_{n}$ is a maximal antichain in $p_{\omega}^{<\infty}$. By induction hypotheses (2), (4) and (5) it follows that $p_{\omega} \in \mathbb{P}$.

For each $n$ and $t \in A_{n}$ and $Y \in \Sigma_{p_{\omega}}(t)$ define $F_{t}(Y)=W_{t-Y}$ and note that $\lambda\left(F_{t}(Y)\right)<J^{*}\left(K_{n}\right)$. Since $\nu\left(\Sigma_{p_{\omega}}(t)\right)=\nu\left(\Sigma_{p_{n}}(t)\right) \geq K_{n}+1$ for $t \in A_{n}$ it follows that if $S_{t}$ is defined to be

$$
\left\{(x, v) \in[0,1]^{k} \times P \mid \nu\left(\left\langle x, v, F_{t}\right\rangle_{K_{n}}\right)<K_{n}\right\},
$$


then $\lambda \times \sigma\left(S_{t}\right) \leq 1 /\left(K_{n}+1\right)$. It follows that

$$
\lambda \times \sigma\left(\bigcup_{n=0}^{\infty} \bigcup_{t \in A_{n}} S_{t}\right) \leq \sum_{n=0}^{\infty} \frac{\left|A_{n}\right|}{K_{n}+1} \leq 1 / 2
$$

by induction hypothesis (9). Therefore it is possible to choose $x^{*} \in[0,1]^{k}$ such that

$$
\sigma\left(\left\{v \in P \mid\left(x^{*}, v\right) \notin \bigcup_{n=0}^{\infty} \bigcup_{t \in A_{n}} S_{t}\right\}\right)>1 / 3
$$

Now refer to Definition 2.1 to choose $v^{*} \in P$ such that

$$
\left(g^{*}, v^{*}\right) \in G \times P \backslash\left(\bigcup_{n=0}^{\infty} \bigcup_{t \in A_{n}} S_{t}\right)
$$

and, using the inequality $\lambda(W)<J(1 / 2,1 / 3)$ of induction hypothesis (6) and the fact that the action $*$ is measure-preserving, such that

$$
\lambda^{s}\left(W \cap\left(g^{*} * \Lambda\left(v^{*}\right)\right)\right) \leq 1 / 2 .
$$

Now let $p^{*} \leq p_{\omega}$ be defined by

$$
p^{*}=\left\{c \in p_{\omega} \mid(\forall n)(\forall m) \text { if } c\left\lceil m \in A_{n}, \text { then } c(m) \in\left\langle x^{*}, v^{*}, F_{c \nmid m}\right\rangle_{K_{n}}\right\}\right.
$$

and note that $p^{*} \in \mathbb{P}$ since $\left(x^{*}, v^{*}\right)$ satisfies condition (2.15). Letting $G$ be the canonical name for the generic real obtained from $\mathbb{P}$, let $g_{n}$ be a name for the unique element of $A_{n}$ such that $g_{n} \subseteq G$ and note that

$$
p^{*} \Vdash " \bigcup_{i=0}^{\infty} B_{i} \subseteq W \cup \bigcup_{n=0}^{\infty} W_{g_{n}} G\left(\left|g_{n}\right|\right) "
$$

by condition (17) and so

$$
\begin{aligned}
p^{*} \Vdash & " \lambda^{s}\left(\left(\Lambda\left(v^{*}\right)+x^{*}\right) \cap\left(\bigcup_{i=0}^{\infty} B_{i}\right)\right) \\
& \leq \sum_{n=0}^{\infty} \lambda^{s}\left(\left(\Lambda\left(v^{*}\right)+x^{*}\right) \cap\left(F_{g_{n}}\left(G\left(\left|g_{n}\right|\right)\right)\right)\right)+\lambda^{s}\left(\left(\Lambda\left(v^{*}\right)+x^{*}\right) \cap W\right) " .
\end{aligned}
$$

Observe that by inequality (2.16) it follows that the last summand in inequality (2.18) is less than $1 / 2$. From Definition (2.17) of $p^{*}$ it follows that $G\left(\left|g_{n}\right|\right) \in$ $\left\langle x^{*}, v^{*}, F_{g_{n}}\right\rangle_{K_{n}}$ for all $n$ and hence

$$
\lambda^{s}\left(\left(\Lambda\left(v^{*}\right)+x^{*}\right) \cap\left(F_{g_{n}}\left(G\left(\left|g_{n}\right|\right)\right)\right)\right)=\Lambda^{F_{g_{n}}\left(G\left(\left|g_{n}\right|\right)\right)}\left(v^{*}, x^{*}\right) \leq 1 / K_{n} .
$$

Therefore

$$
p^{*} \Vdash " \lambda^{s}\left(\left(\Lambda\left(v^{*}\right)+x^{*}\right) \cap\left(\bigcup_{i=0}^{\infty} B_{i}\right)\right) \leq \sum_{i=0}^{\infty} 1 / K_{i}+1 / 2<1 "
$$

by induction hypothesis (9). It follows from the hypothesis of Definition (2.1) that $\lambda^{s}\left(\Lambda\left(v^{*}\right)\right) \geq 1$ and therefore the fact that $p^{*} \Vdash " \Lambda\left(v^{*}\right)+x^{*} \nsubseteq \bigcup_{i=0}^{\infty} B_{i}$ " contradicts that $\left(x^{*}, v^{*}\right)$ belongs to $V$. 
In order to see that the induction can be carried out, begin by letting $K_{0}=2$ so that induction hypothesis (9) is satisfied. Next, choose $p_{0}^{1} \leq p$ and $N_{0}$ such that

$$
p_{0}^{1} \Vdash \text { “ } \sum_{i=N_{0}+1}^{\infty} r_{i}^{k}<J^{*}\left(K_{0}\right) " .
$$

Then choose $p_{0}^{2} \leq p_{0}^{1}$ such that $p_{0}^{2} \Vdash$ " $\bigcup_{i=0}^{N_{0}} B_{i}=\check{W}$ " for some open set $W$. Let $t \in\left(p_{0}^{2}\right)<\infty$ be such that $\nu\left(\Sigma_{p_{0}^{2}}(t)\right)>K_{0}+1$ and let $p_{0}=p_{0}^{2} \cap[t]$ and let $A_{0}=\{t\}$. Induction hypothesis (6) is then satisfied. Induction hypotheses (44), (17) and (18) are not relevant at this stage.

Now suppose that $N_{n}, p_{n}, K_{n}$ and $\left\{A_{n}\right\}_{i=0}^{n}$ have been constructed satisfying the induction hypotheses and select $K_{n+1} \geq n+1$ so large that

$$
\sum_{i=0}^{n} \frac{\left|A_{i}\right|}{K_{i}+1}+\frac{\sum_{t \in A_{n}}\left|\Sigma_{p_{n}}(t)\right|}{K_{n+1}+1}<1 / 2
$$

Choose $p_{n}^{1} \leq p_{n}$ such that for each $t \in A_{n}$ and $Y \in \Sigma_{p_{n}}(t)$ there is some $N_{t \frown Y}$ such that

$$
p_{n}^{1} \cap\left[t^{\frown} Y\right] \Vdash " \sum_{i=N_{t} \frown Y}^{\infty} r_{i}^{k}<J^{*}\left(K_{n+1}\right) ”
$$

and let $N_{n+1}=\max \left\{N_{t-Y} \mid t \in A_{n}\right.$ and $\left.Y \in \Sigma_{p_{n}}(t)\right\}$. Then choose $p_{n}^{2} \leq p_{n}^{1}$ such that for each $t \in A_{n}$ and $Y \in \Sigma_{p_{n}}(t)$ there is an open set $W_{t-Y}$ such that

$$
p_{n}^{2} \cap[t \frown Y] \Vdash \text { " } \bigcup_{i=N_{n}}^{N_{n+1}} B_{i}=\check{W}_{t \frown Y} \text { ". }
$$

For each $t \in A_{n}$ and $Y \in \Sigma_{p_{n}}(t)$ select $\tau(t, Y) \in\left(p_{n}^{2}\right)^{<\infty}$ such that $t^{\frown} Y \subseteq \tau(t, Y)$ and $\nu\left(\Sigma_{p_{n}^{2}}(\tau(t, Y))\right) \geq K_{n+1}+1$ and such that $|\tau(t, Y)|>n+1$ so that induction hypothesis (3) will hold if $A_{n+1}$ is defined to be $\left\{\tau(t, Y) \mid t \in A_{n}\right.$ and $\left.Y \in \Sigma_{p_{n}}(t)\right\}$. Then let

$$
p_{n+1}=\bigcup\left\{p_{n}^{2} \cap[\tau(t, Y)] \mid t \in A_{n} \text { and } Y \in \Sigma_{p_{n}}(t)\right\}
$$

guaranteeing that induction hypothesis (5) holds.

To see that induction hypothesis (8) holds use condition (2.20). Condition (2.19) guarantees that induction hypothesis (9) holds and everything else is immediate from the construction.

Finally, no restriction will be imposed on $\kappa$. It suffices to assume that $\kappa=\omega$. For $p \in \mathbb{P}_{\omega}, n \in \omega$ and $\theta: n \rightarrow T^{<\infty}$ define

$$
p[\theta]= \begin{cases}p(j) & \text { if } j \geq n, \\ p(j) \cap[\theta(j)] & \text { if } j \in n,\end{cases}
$$

noting, of course, that $p[\theta]$ may not belong to $\mathbb{P}_{\omega}$ in general.

If the theorem is false, then, as in the previous cases, there is $p \in \mathbb{P}_{\omega}$ such that

$$
p \Vdash_{\mathbb{P}_{\omega}} \text { " } \bigcup_{x \in V \cap[0,1]^{k}} \bigcup_{v \in V \cap P}(\Lambda(v)+x) \subseteq \bigcup_{i=0}^{\infty} B_{i} \text { and } \sum_{i=0}^{\infty} r_{i}^{k}<J(1 / 2,1 / 3) ",
$$

where, as before, $B_{i}$ is the cube $\prod_{j=1}^{k}\left(q_{j, i}, q_{j, i}+r_{i}\right)$. 
Fix a function $e: \mathbb{N} \rightarrow \mathbb{N}$ such that the pre-image of each integer under $e$ is infinite and such that $e(i) \leq i$ for each $i \in \mathbb{N}$. Now construct by induction on $n$ conditions $p_{n} \in \mathbb{P}_{\omega}$, integers $N_{n}$ and $K_{n} \geq 1$ and $A_{n}^{e(i)} \subseteq T^{<\infty}$ for $i \leq n$ such that:

(1) $p_{0} \leq p$ and $p_{n+1} \leq p_{n}$ for each $n$.

(2) $\{[t]\}_{t \in A_{n}^{i}}$ is a finite open cover of $p_{n}(i)$ by disjoint non-empty sets if $A_{n}^{i}$ is defined.

(3) If $0 \leq i \leq n$ and $i \neq e(n+1)$, then $A_{n}^{i}=A_{n+1}^{i}$ If $A_{n}^{i}$ is defined.

(4) If $t \in A_{n}^{e(n)}$, then $|t|>n$.

(5) $\Sigma_{p_{n}(i)}(t)=\Sigma_{p_{m}(i)}(t)$ for each $m \geq n$ and for each $t \in A_{n}^{i}$. As a consequence of this it is possible to define $\Sigma\left(A_{n}^{i}\right)=\left\{t \frown X \mid t \in A_{n}^{i}\right.$ and $X \in$ $\left.\Sigma_{p_{n}(i)}(t)\right\}$ and have that $\Sigma\left(A_{n}^{i}\right)=\left\{t \frown X \mid t \in A_{n}^{i}\right.$ and $\left.X \in \Sigma_{p_{m}(i)}(t)\right\}$ for any $m \geq n$.

(6) $\nu\left(\Sigma_{p_{n}(e(n))}(t)\right) \geq K_{n}+1 \geq n$ for each $t \in A_{n}^{e(n)}$.

(7) There is an open set $W$ such that $p_{0} \Vdash$ " $\bigcup_{i=0}^{N_{0}} B_{i}=\check{W} "$.

(8) For each $\theta \in \prod_{i=0}^{n} \Sigma\left(A_{n}^{i}\right)$ there is an open set $W_{\theta}$ such that $p_{n+1}[\theta] \Vdash$ " $\bigcup_{i=N_{n}+1}^{N_{n+1}} B_{i}=\check{W}_{\theta}$ " using the convention that $\Sigma\left(A_{n}^{i}\right)=\{\emptyset\}$ in case $A_{n}^{i}$ is not defined.

(9) $p_{n} \Vdash " \prod_{i=0}^{e(n)-1}\left|\Sigma\left(A_{n}^{i}\right)\right| \prod_{i=e(n)+1}^{n}\left|\Sigma\left(A_{n}^{i}\right)\right| \sum_{i=N_{n}+1}^{\infty} r_{i}^{k}<J^{*}\left(K_{n}\right) "$.

(10) $\sum_{i=0}^{n} \frac{2\left|A_{i}^{e(i)}\right|}{K_{i}}<1 / 2$.

Given that this can be done, define $p_{\omega}$ by setting $p_{\omega}(i)=\bigcap_{n=0}^{\infty} p_{n}(i)$ and note that each $A_{n}^{i}$ is a maximal antichain in $p_{\omega}(i)$. As before, the induction hypotheses and the choice of the enumerating function $e$ yield that $p_{\omega}(i) \in \mathbb{P}$ for each $i$ and so $p_{\omega} \in \mathbb{P}_{\omega}$

For each $n$ and $t \in A_{n}^{e(n)}$ and $Y \in \Sigma_{p_{\omega}(e(n))}(t)$ and

$$
\theta \in \prod_{i=0}^{e(n)-1} \Sigma\left(A_{n}^{i}\right) \times \prod_{i=e(n)+1}^{n} \Sigma\left(A_{n}^{i}\right)
$$

define $\theta *(t, Y) \in \prod_{i=0}^{n} \Sigma\left(A_{n}^{i}\right)$ by

$$
\theta *(t, Y)(i)= \begin{cases}\theta(i) & \text { if } i \neq e(n+1), \\ t^{\frown} Y & \text { if } i=e(n+1)\end{cases}
$$

and then define

$$
F_{t}(Y)=\bigcup\left\{W_{\theta *(t, Y)} \mid \theta \in \prod_{i=0}^{e(n)-1} \Sigma\left(A_{n}^{i}\right) \times \prod_{i=e(n)+1}^{n} \Sigma\left(A_{n}^{i}\right)\right\}
$$

and note that $\lambda\left(F_{t}(Y)\right)<J^{*}\left(K_{n}\right)$.

Since $\nu\left(\Sigma_{p_{\omega}(e(n))}(t)\right)=\nu\left(\Sigma_{p_{n}(e(n))}(t)\right) \geq K_{n}+1$ for $t \in A_{n}^{e(n)}$ it follows that if $S_{t}$ is defined by expression (2.13) then $\lambda \times \sigma\left(S_{t}\right) \leq 1 /\left(K_{n}+1\right)$ and the proof then proceeds as in the previous cases. 


\section{AN INTERMEDIATE NORM}

In order to establish the validity of hypothesis (2.12) an intermediate family of norms will be introduced and an inequality will be proved between these new norms and those of 92 , It will then be shown in 94 that the analogue of hypothesis (2.12) holds for these new norms. This will be enough to conclude that hypothesis (2.12) itself holds. For the next definition recall notation 2.1.

Definition 3.1. Let $\left(Q_{1}, \mu_{1}\right)$ and $\left(Q_{2}, \mu_{2}\right)$ be probability spaces and $\gamma>0$. For $A$ and $B$ measurable subsets of $\left(Q_{1} \times Q_{2}\right)^{k}$ define $A \Subset_{\gamma} B$ by induction on the integer $k$. If $k=1$, then $A \Subset_{\gamma} B$ if and only if $A \subseteq B$ and either

$$
\left(\exists p \in Q_{1}\right) B\langle p\rangle \neq \emptyset \text { and } \mu_{2}(B\langle p\rangle \backslash A\langle p\rangle)<\gamma
$$

or

$$
\mu_{1}\left(\left\{p \in Q_{1} \mid A\langle p\rangle=\emptyset \neq B\langle p\rangle\right\}\right)<\gamma .
$$

If $k>1$, then $A \Subset_{\gamma} B$ if and only if $A \subseteq B$ and

$$
\left\{x \in Q_{1} \times Q_{2} \mid A\langle x\rangle \Subset_{\gamma} B\langle x\rangle \neq \emptyset\right\} \Subset_{\gamma} \pi(B),
$$

where $\pi(B)=\left\{x \in Q_{1} \times Q_{2} \mid B\langle x\rangle \neq \emptyset\right\}$. Note that the first use of $\Subset_{\gamma}$ uses the inductive hypothesis for the $(k-1)$-fold product.

In the case $k=1$ it is useful to think of the relation $A \Subset_{\gamma} B$ as saying that either $A$ contains all but a set of measure $\gamma$ of a vertical section of $B$ or there is a function contained in $A$ whose domain is all but a set of measure $\gamma$ of the domain of $B$. If $k>1$, then the definition of $A \Subset_{\gamma} B$ is more complicated because of the Fubini type product used in the inductive definition.

Definition 3.2. Given $M \in \mathbb{N}, \gamma>0, \xi>0$ and $\mathcal{Y} \subseteq \mathcal{P}_{\xi}(M)$ define a set $\Theta_{\gamma}(\mathcal{Y}) \subseteq \mathbb{N}$ by defining which $j \in \mathbb{N}$ belong to $\Theta_{\gamma}(\mathcal{Y})$.

- $0 \in \Theta_{\gamma}(\mathcal{Y})$ if and only if $\mathcal{Y} \neq \emptyset$.

- $1 \in \Theta_{\gamma}(\mathcal{Y})$ if and only if $\bigcup \mathcal{Y}=M$.

- If $j \geq 1$, then $j+1 \in \Theta_{\gamma}(\mathcal{Y})$ if and only if for every pair of finite probability spaces $\left(Q_{1}, \mu_{1}\right)$ and $\left(Q_{2}, \mu_{2}\right)$ and any $A \subseteq\left(Q_{1} \times Q_{2}\right)^{j}$ and $\Psi: A \rightarrow M$ there is $Y \in \mathcal{Y}$ such that $\Psi^{-1} Y \Subset_{\gamma} A$.

Define $\rho_{\gamma}(\mathcal{Y})=j$ if $j+1$ is the least integer that does not belong to $\Theta_{\gamma}(\mathcal{Y})$. Observe that if $\mathcal{Y}_{1} \subseteq \mathcal{Y}_{2}$, then $\Theta_{\gamma}\left(\mathcal{Y}_{1}\right) \leq \Theta_{\gamma}\left(\mathcal{Y}_{2}\right)$ and if $j+1 \in \Theta_{\gamma}(\mathcal{Y})$, then $j \in \Theta_{\gamma}(\mathcal{Y})$.

For technical reasons, a superset of $\Theta_{\gamma}(\mathcal{Y})$ will be needed. If $\delta>0$ and $L \in \mathbb{N}$, then a probability space will be said to be $(\delta, L)$-fine if and only if every set of measure greater than $\delta$ has cardinality at least $L$. $\Theta_{\gamma}^{\delta, L}(\mathcal{Y})$ is defined the same way as $\Theta_{\gamma}(\mathcal{Y})$ except that if $j \geq 1$, then $j+1 \in \Theta_{\gamma}^{\delta, L}(\mathcal{Y})$ if and only if for every pair of finite $(\delta, L)$-fine probability spaces $\left(Q_{1}, \mu_{1}\right)$ and $\left(Q_{2}, \mu_{2}\right)$ and any $A \subseteq\left(Q_{1} \times Q_{2}\right)^{j}$ and $\Psi: A \rightarrow M$ there is $Y \in \mathcal{Y}$ such that $\Psi^{-1} Y \Subset_{\gamma} A$.

Observe that, while there are similarities to $\Theta$ of Definition 2.4 there is a crucial difference: The definition of $\Theta(\mathcal{Y})$ is inductive while the definition of $\Theta_{\gamma}(\mathcal{Y})$ is not. (Of course, this relies on the inductive definition of $\Subset_{\gamma}$.) Nevertheless, the two sets are linked, as will be seen in the next result, Lemma 3.3. Keep in mind that $\Lambda, J, K$ and $\{\epsilon(i)\}_{i=0}^{\infty}$ are still fixed as in $\Upsilon_{2}$ Before proceeding to the proof of Lemma 3.3 the observation that Definitions 3.1 and 3.2 have equivalent martingale versions will turn out to be useful. 
Definition 3.3. For a fixed integer $k$ recall that a martingale is a family of finite probability spaces indexed by sequences of length less than or equal to $k$ and denoted $\mathfrak{M}(t)=\left(M_{t}, \sigma_{t}\right)$ for $t$ a sequence of length less than or equal to $k$. The integer $k$ will be called the length of the martingale. (For the purposes of this section, $\mathfrak{M}(t)$ will always be a product of two spaces, but this can be suppressed for the moment.) The probability space $S(\mathfrak{M})$ is defined by induction on $k$.

If $k=0$ the space is $S(\mathfrak{M})=\mathfrak{M}(\emptyset)$. If $k>0$, then define $\mathfrak{M}_{x}(t)=\mathfrak{M}(x \frown t)$ for $t$ of length less than $k$. Then $S(\mathfrak{M})=\left(M_{\mathfrak{M}}, \sigma_{\mathfrak{M}}\right)$, where $M_{\mathfrak{M}}$ is the set of pairs $(x, y)$ such that $x \in M_{\emptyset}$ and $y \in M_{\mathfrak{M}_{x}}$ with the measure $\sigma_{\mathfrak{M}}$ defined by

$$
\sigma_{\mathfrak{M}}(X)=\sum_{x \in \mathfrak{M}(\emptyset)} \sigma_{\emptyset}(\{x\}) \sigma_{\mathfrak{M}_{x}}(X\langle x\rangle)
$$

for $X \subseteq M_{\mathfrak{M}}$.

Definition 3.4. Let a martingale of length $k$ of products of probability spaces and denoted by $\mathfrak{M}(t)=\left(M_{t}, \sigma_{t}\right)$ be given. For $A$ and $B$ measurable subsets of $M_{\mathfrak{M}}$ define $A \Subset_{\gamma}^{\mathfrak{M}} B$ by induction on the integer $k$. If $k=0$, then $A \Subset_{\gamma}^{\mathfrak{M}} B$ is defined exactly as in Definition 3.1. If $k \geq 1$, then $A \Subset_{\gamma}^{\mathfrak{M}} B$ if and only if $A \subseteq B$ and

$$
\left\{x \in M_{\emptyset} \mid A\langle x\rangle \Subset_{\gamma}^{\mathfrak{M}_{x}} B\langle x\rangle \neq \emptyset\right\} \Subset_{\gamma} \pi(B),
$$

where $\pi(B)$ is defined in analogy with Definition 3.1 and the notation $A\langle x\rangle$ is now used to denote all $t \in M_{\mathfrak{M}}$ such that $t(0)=x$.

Definition 3.5. Just as in Definition 3.2 let $\bar{M} \in \mathbb{N}, \gamma>0, \xi>0$ and $\mathcal{Y} \subseteq \mathcal{P}_{\xi}(\bar{M})$ be given and define a set $\Theta_{\gamma}^{+}(\mathcal{Y}) \subseteq \mathbb{N}$ by defining which $j \in \mathbb{N}$ belong to $\Theta_{\gamma}^{+}(\mathcal{Y})$. The definition for 0 and 1 is exactly the same as in Definition 3.2. If $j \geq 1$, then $j+1 \in \Theta_{\gamma}(\mathcal{Y})$ if and only if for every martingale $\mathfrak{M}$ of length $j$ of products of pairs of probability spaces and any $A \subseteq M_{\mathfrak{M}}$ and $\Psi: A \rightarrow \bar{M}$ there is $Y \in \mathcal{Y}$ such that $\Psi^{-1} Y \Subset_{\gamma}^{\mathfrak{M}} A$. A version of this definition restricted to martingales consisting of $(\delta, L)$-fine probability spaces will also be needed.

Lemma 3.1. For any $\bar{M} \in \mathbb{N}, \gamma>0, \xi>0$ and $\mathcal{Y} \subseteq \mathcal{P}_{\xi}(\bar{M})$ the sets $\Theta_{\gamma}(\mathcal{Y})$ and $\Theta_{\gamma}^{+}(\mathcal{Y})$ are the same.

Proof. Since products are simple martingales, it suffices to show that $\Theta_{\gamma}(\mathcal{Y}) \supseteq$ $\Theta_{\gamma}^{+}(\mathcal{Y})$. Given a martingale $\mathfrak{M}$ of length $j-1$ and $A \subseteq M_{\mathfrak{M}}$ and $\Psi: A \rightarrow \bar{M}$ let $\mathfrak{M}(t)$ be the product of the two probability spaces $\left(Q_{0}^{t}, \sigma_{0}^{t}\right)$ and $\left(Q_{1}^{t}, \sigma_{1}^{t}\right)$. Let $D$ consist of all $t\left\lceil n\right.$ for some $t \in M_{\mathfrak{M}}$ and $n<j$. For $i \in 2$ let

$$
\left(Q_{i}^{*}, \sigma_{i}^{*}\right)=\prod_{t \in D}\left(Q_{i}^{t}, \sigma_{i}^{t}\right)
$$

and then define a mapping $R: \prod_{n \in j} Q_{0}^{*} \times Q_{1}^{*} \rightarrow M_{\mathfrak{M}}$ by $R(x)(n)=x(n)(R(x)\lceil n)$ noting that this is an inductive definition and that $x(n)$ is a function with domain $D$ and $R(x)\lceil n \in D$.

Let $A^{*}=R^{-1} A$ and define $\Psi^{*}: A^{*} \rightarrow \bar{M}$ by $\Psi^{*}(x)=\Psi(R(x))$. A direct verification establishes that $\left(\Psi^{*}\right)^{-1} Y \Subset_{\gamma} A^{*}$ if and only if $\Psi^{-1} Y \Subset_{\gamma}^{\mathfrak{M}} A$ for $Y \in$ $\mathcal{Y}$.

The following technical fact can be established by routine arguments. 
Lemma 3.2. If $\left(Q_{0}, \sigma_{0}\right)$ and $\left(Q_{1}, \sigma_{1}\right)$ are Polish probability spaces, $\epsilon>0$ and $A \subseteq$ $Q_{0} \times Q_{1}$ is measurable of positive measure and $\zeta<1$, then there are $\left\{A_{j}^{0} \times A_{j}^{1}\right\}_{j=1}^{k}$ such that

(1) each $A_{j}^{i}$ is a measurable subset of $Q_{i}$,

(2) if $j \neq i$, then $A_{j}^{0} \cap A_{i}^{0}=\emptyset$,

(3) $\sigma_{0} \times \sigma_{1}\left(A \backslash \bigcup_{j=1}^{k} A_{j}^{0} \times A_{j}^{1}\right)<\epsilon$,

(4) $\frac{\sigma_{1}\left(A\langle x\rangle \cap A_{j}^{1}\right)}{\sigma_{1}\left(A_{j}^{1}\right)}>\zeta$ for each $j$ and $x \in A_{j}^{0}$.

Proof. Regularity of the measures and Fubini's Theorem yield a compact set $C \subseteq A$ and an open set $U \supseteq A$ such that $\sigma_{0} \times \sigma_{1}(U \backslash C)$ is so small that

$$
\sigma_{0}\left(\left\{q \in Q_{0} \mid \sigma_{1}(C\langle q\rangle) / \sigma_{1}(U\langle q\rangle)<\zeta\right\}\right)<\epsilon / 2 .
$$

Let $\left\{B_{n}\right\}_{n \in \omega}$ enumerate a base for $Q_{0} \times Q_{1}$ consisting of rectangular sets and let $U_{n}=\bigcup\left\{B_{j} \mid B_{j} \subseteq U\right.$ and $\left.j \leq n\right\}$ and choose $k$ large enough that $C \subseteq U_{k}$ still holds. Since there are only finitely many sets that can be formed by the first $k$ basic open sets, the result follows.

Lemma 3.3. If $j \in \mathbb{N}$ and $\gamma<\min \left(\epsilon(j), K^{q^{\prime}} j^{q^{\prime}} J^{*}(j)^{q^{\prime} / q}\right)$ and $\mathcal{Y} \subseteq \mathcal{P}(M)$, then $\Theta_{\gamma}(\mathcal{Y}) \subseteq \Theta(\mathcal{Y})$. Indeed, $\Theta_{\gamma}^{\delta, L}(\mathcal{Y}) \subseteq \Theta(\mathcal{Y})$ for any $\delta>0$ and $L$.

Proof. Proceed by induction on $j$ to show that if $j \in \Theta_{\gamma}(\mathcal{Y})$, then $j \in \Theta(\mathcal{Y})$, the cases $j=0$ and $j=1$ being trivial.

Now suppose that $2 \notin \Theta(\mathcal{Y})$; in other words, there is a function $F$ from $\mathcal{Y}$ to the open subsets of $\mathbb{R}^{k}$ of measure less than $J^{*}(1)$ such that, letting

$$
A=\left\{(x, p) \in[0,1]^{k} \times P \mid 1 \notin \Theta\left(\langle x, p, F\rangle_{1}\right)\right\},
$$

it follows that $\lambda \times \sigma(A)>1 / 2$. Recall from Lemma 2.1 that the mapping $\Lambda^{F(Y)}$ is measurable for each $Y \in \mathcal{Y}$. Hence it is possible to find a measurable $A^{\prime} \subseteq A$ such that $\lambda \times \sigma\left(A^{\prime}\right)>1 / 2$ and each of these mappings is continuous on $A^{\prime}$.

For each $(x, p) \in A^{\prime}$ it follows that there is some $a \in M \backslash \bigcup\langle x, p, F\rangle_{1}$. Note that $Y \notin\langle x, p, F\rangle_{1}$ if and only if $\Lambda^{F(Y)}(p, x)>1$ and so there is an open rectangle $U_{1}(Y) \times U_{0}(Y)$ which is a neighbourhood of $(x, p)$ such that $\Lambda^{F(Y)}\left(p^{\prime}, x^{\prime}\right)>1$ for all $\left(x^{\prime}, p^{\prime}\right) \in U_{1}(Y) \times U_{0}(Y) \cap A^{\prime}$. Hence, letting $U_{i}=\bigcap\left\{U_{i}(Y) \mid a \in Y \in \mathcal{Y}\right\}$, there is a neighbourhood $U_{1} \times U_{0}$ of $(x, p)$ such that $a \notin \bigcup\left\langle x^{\prime}, p^{\prime}, F\right\rangle_{1}$ for all $\left(x^{\prime}, p^{\prime}\right) \in$ $U_{1} \times U_{0} \cap A^{\prime}$. It follows from applying Lemma 3.2 to sufficiently many of these neighbourhoods that there are pairwise disjoint measurable rectangles $\left\{U_{1}^{j} \times U_{0}^{j}\right\}_{j=1}^{m}$ such that:

(1) for each $j$ there is $a_{j} \in M$ such that $a_{j} \notin \bigcup\langle x, p, F\rangle_{1}$ for all $(x, p) \in$ $U_{1}^{j} \times U_{0}^{j} \cap A^{\prime}$

(2) $\lambda \times \sigma\left(A^{\prime} \cap \bigcup_{j=1}^{m}\left(U_{1}^{j} \times U_{0}^{j}\right)\right)>1 / 2$,

(3) $\sigma_{0}\left(A^{\prime}\langle x\rangle \cap U_{j}^{0}\right)>\sigma_{0}\left(U_{j}^{0}\right) \zeta$ for each $j$ and $x \in U_{j}^{1}$, where $\zeta<1$ is sufficiently large that $(2 \epsilon(1)-\gamma) \zeta>\epsilon(1)$.

For the last assertion use the assumption on $\gamma$ that $\gamma<\epsilon(1)$.

Now let $\mathcal{M}_{1}$ be the finite Boolean algebra of subsets of $[0,1]^{k}$ generated by $\left\{U_{1}^{j}\right\}_{j=1}^{m}$ and let $\mathcal{M}_{0}$ be the finite Boolean algebra of subsets of $P$ generated by $\left\{U_{0}^{j}\right\}_{j=1}^{m}$. Let $Q_{i}$ be the set of all atoms of $\mathcal{M}_{i}$. Let $\mu_{0}$ be the measure defined on the subsets of $Q_{0}$ by assigning $\mu_{0}(X)=\sum_{a \in X} \sigma(a)$ and let $\mu_{1}$ be the measure 
defined on the subsets of $Q_{1}$ by assigning $\mu_{1}(X)=\sum_{a \in X} \lambda(a)$ so that both $\left(Q_{0}, \mu_{0}\right)$ and $\left(Q_{1}, \mu_{1}\right)$ are finite probability spaces. If they are not $(\delta, L)$-fine, use the fact that $P$ is atomless to refine the atoms so that $\left(Q_{0}, \mu_{0}\right)$ and $\left(Q_{1}, \mu_{1}\right)$ are $(\delta, L)$-fine. Let

$$
A^{*}=\left\{q_{1} \in Q_{1} \mid \mu_{0}\left(\left\{q_{0} \in Q_{0} \mid(\exists j \leq m) q_{1} \times q_{0} \subseteq U_{1}^{j} \times U_{0}^{j}\right\}\right)>2 \epsilon(1)\right\}
$$

and note that $\mu_{1}\left(A^{*}\right)>2 K^{q^{\prime}} J^{*}(1)^{q^{\prime} / q}$ by Definition 2.3 since $\left(Q_{0}, \mu_{0}\right)$ and $\left(Q_{1}, \mu_{1}\right)$ are probability spaces. Let $\bar{A}=\left\{\left(q_{1}, q_{0}\right) \mid q_{1} \in A^{*}\right.$ and $\left.(\exists j \leq m) q_{1} \times q_{0} \subseteq U_{1}^{j} \times U_{0}^{j}\right\}$. It is then possible to define $\Psi: \bar{A} \rightarrow M$ such that for each $\left(q_{1}, q_{0}\right) \in \bar{A}$ there is some $j$ such that $q_{1} \times q_{0} \subseteq U_{1}^{j} \times U_{0}^{j}$ and $\Psi\left(q_{1}, q_{0}\right)=a_{j}$. Since $\rho_{\gamma}(\mathcal{Y}) \geq 2$ it is possible to find $Y \in \mathcal{Y}$ such that $\Psi^{-1} Y \Subset_{\gamma} \bar{A}$. There are now two cases to consider.

Case One. There is some $q_{1} \in A^{*}$ such that $\mu_{0}\left(\left(\bar{A} \backslash\left(\Psi^{-1} Y\right)\right)\left\langle q_{1}\right\rangle\right)<\gamma$.

Since $\mu_{0}(\bar{A}\langle q\rangle)>2 \epsilon(1)$ for each $q \in A^{*}$ it follows that $\mu_{0}\left(\left(\Psi^{-1} Y\right)\left\langle q_{1}\right\rangle\right)>2 \epsilon(1)-$ $\gamma$. If $q_{0} \in\left(\Psi^{-1} Y\right)\left\langle q_{1}\right\rangle$, then $\Psi\left(q_{1}, q_{0}\right) \in Y$ and so $Y$ does not belong to $\langle x, p, F\rangle_{1}$ for any $(x, p) \in A^{\prime}$ such that $x \in q_{1}$ and $p \in q_{0}$. Fix some $x^{*} \in q_{1}$. Therefore $\Lambda^{F(Y)}\left(p, x^{*}\right)>1$ for every $p \in \bigcup\left(\Psi^{-1} Y\right)\left\langle q_{1}\right\rangle \cap A^{\prime}\left\langle x^{*}\right\rangle$. Using the fact that the $U_{1}^{j} \times$ $U_{0}^{j}$ are pairwise disjoint, it follows that $\sigma\left(\bigcup\left(\Psi^{-1} Y\right)\left\langle x^{*}\right\rangle \cap A^{\prime}\left\langle x^{*}\right\rangle\right)>(2 \epsilon(1)-\gamma) \zeta>$ $\epsilon(1)$ and so $\lambda(F(Y))>J^{*}(1)$ by Definition 2.1. This contradicts the hypothesis on $F$ that the measure of $F(Y)$ is less than $J^{*}(1)$.

Case Two. $\mu_{1}\left(\left\{q_{1} \in Q_{1} \mid\left(\Psi^{-1} Y\right)\left\langle q_{1}\right\rangle=\emptyset \neq \bar{A}\left\langle q_{1}\right\rangle\right\}\right)<\gamma$.

Observe that $q_{1} \in A^{*}$ if and only if $\emptyset \neq \bar{A}\left\langle q_{1}\right\rangle$. Therefore

$$
\mu_{1}\left(\left\{q_{1} \in A^{*} \mid\left(\Psi^{-1} Y\right)\left\langle q_{1}\right\rangle=\emptyset\right\}\right)<\gamma<K^{p} J^{*}(1)^{p / q}
$$

and so $\mu_{1}\left(\left\{q_{1} \in A^{*} \mid\left(\Psi^{-1} Y\right)\left\langle q_{1}\right\rangle \neq \emptyset\right\}\right)>\mu_{1}\left(A^{*}\right)-K^{p} J^{*}(1)^{p / q}>K^{p} J^{*}(1)^{p / q}$. From inequality (2.7) with $\epsilon=1$ and the fact that the measure of $F(Y)$ is less than $J^{*}(1)$ it follows that it is possible to choose $x^{*} \in q_{1}^{*} \in A^{*}$ so that $\left(\Psi^{-1} Y\right)\left\langle q_{1}^{*}\right\rangle \neq \emptyset$ and such that $\Lambda^{F(Y)}\left(p, x^{*}\right)<1$ for all $p \in P$. Choose any $q_{0}^{*} \in\left(\Psi^{-1} Y\right)\left\langle q_{1}^{*}\right\rangle$ and $p^{*} \in q_{0}^{*}$ such that $\left(x^{*}, p^{*}\right) \in A^{\prime}$ and note that then $\Psi\left(q_{1}^{*}, q_{0}^{*}\right) \in Y$. On the other hand, it follows that $\Lambda^{F(Y)}\left(p^{*}, x^{*}\right)<1$ by the choice of $x^{*}$ and so $Y \in\left\langle x^{*}, p^{*}, F\right\rangle_{1}$ and hence, since $\left(x^{*}, p^{*}\right) \in A^{\prime}, \Psi\left(q_{1}^{*}, q_{0}^{*}\right) \notin Y$. This contradiction finishes the argument for the case $j=2$.

Now assume that $j \geq 2$ and that $j+1 \in \Theta_{\gamma}(\mathcal{Y})$. Let $F$ be a function from $\mathcal{Y}$ to the open subsets of $\mathbb{R}^{k}$ of measure less than $J^{*}(j)$. As in the initial case, let

$$
A=\left\{(x, p) \in[0,1]^{k} \times P \mid j \notin \Theta\left(\langle x, p, F\rangle_{j}\right)\right\}
$$

and, aiming for a contradiction, suppose that $\lambda \times \sigma(A)>1 /(j+1)$. Furthermore, it may be assumed that the mapping $\Lambda^{F(Y)}$ is continuous on $A$ for each $Y \in \mathcal{Y}$. From the induction hypothesis it follows from applying Lemma 3.2 that there are pairwise disjoint measurable rectangles $\left\{U_{1}^{n} \times U_{0}^{n}\right\}_{n=1}^{m}$ such that:

(1) for each $n$ there are finite probability spaces $\left(Q_{0}, \mu_{0}\right)$ and $\left(Q_{1}, \mu_{1}\right)$ and $B \subseteq\left(Q_{0} \times Q_{1}\right)^{j-1}$ and $\Psi: B \rightarrow M$ witnessing that $j \notin \Theta_{\gamma}\left(\langle x, p, F\rangle_{j}\right)$ if $(x, p) \in A \cap U_{1}^{n} \times U_{0}^{n}$ in that $\Psi^{-1} Y \notin_{\gamma} B$ for each $Y \in\langle x, p, F\rangle_{j}$,

(2) $\lambda \times \sigma\left(A \cap \bigcup_{j=1}^{m} U_{1}^{j} \times U_{0}^{j}\right)>1 / 2$,

(3) $\sigma_{0}\left(A\langle x\rangle \cap U_{0}^{j}\right)>\sigma_{0}\left(U_{0}^{j}\right) \zeta$ for each $j$ and $x \in U_{1}^{j}$ where $\zeta<1$ is sufficiently large that $(2 \epsilon(j)-\gamma) \zeta>\epsilon(j)$. 
As in the initial case, let $\mathcal{M}_{1}$ be the finite Boolean algebra of measurable subsets of $[0,1]^{k}$ generated by $\left\{U_{1}^{n}\right\}_{n=1}^{m}$ and $\mathcal{M}_{0}$ be the finite Boolean algebra of measurable subsets of $P$ generated by $\left\{U_{0}^{n}\right\}_{n=1}^{m}$. Letting $Q_{i}$ be the atoms of $\mathcal{M}_{i}$, if $\tilde{A}$ is defined to be the set of all $\left(q_{1}, q_{0}\right) \in Q_{1} \times Q_{0}$ such that there exist finite probability spaces $\left(Q_{0}^{q_{1}, q_{0}}, \mu_{0}^{q_{1}, q_{0}}\right)$ and $\left(Q_{1}^{q_{1}, q_{0}}, \mu_{1}^{q_{1}, q_{0}}\right)$ and $B^{q_{1}, q_{0}} \subseteq\left(Q_{0}^{q_{1}, q_{0}} \times Q_{1}^{q_{1}, q_{0}}\right)^{j-1}$ as well as a function $\Psi^{q_{1}, q_{0}}: B^{q_{1}, q_{0}} \rightarrow M$ such that

$$
\left(\forall(x, q) \in\left(q_{1}, q_{0}\right) \cap A\right)\left(\forall Y \in\langle x, p, F\rangle_{j}\right)\left(\Psi^{q_{0}, q_{1}}\right)^{-1} Y \notin_{\gamma} B^{q_{0}, q_{1}},
$$

then $\mu_{1} \times \mu_{0}(\tilde{A})>1 /(j+1)$ where each $\mu_{i}$ is the measure defined on $Q_{i}$ as in the initial case. Let

$$
A^{*}=\left\{q_{1} \in Q_{1} \mid \mu_{0}\left(\tilde{A}\left\langle q_{1}\right\rangle\right)>2 \epsilon(j)\right\}
$$

and note that $\mu_{1}\left(A^{*}\right)>2 j^{q^{\prime}} K^{q^{\prime}} J^{*}(j)^{q^{\prime} / q}$. Let $\bar{A}=\left\{\left(q_{1}, q_{0}\right) \in \tilde{A} \mid q_{1} \in A^{*}\right\}$.

Note that defining the martingale $\mathfrak{M}$ by setting $\mathfrak{M}(\emptyset)=\left(Q_{1} \times Q_{0}, \mu_{1} \times \mu_{0}\right)$ and, if $t \neq \emptyset$, setting

$$
\mathfrak{M}(t)=\left(Q_{1}^{q_{1}, q_{0}} \times Q_{0}^{q_{1}, q_{0}}, \mu_{1}^{q_{1}, q_{0}} \times \mu_{0}^{q_{1}, q_{0}}\right),
$$

where $t(0)=\left(q_{1}, q_{0}\right)$ satisfies Definition 3.4 and has length $j$. Let $B$ consist of all $\left(q_{0}, q_{1}\right) \frown t \in M_{\mathfrak{M}}$ such that $t \in B^{q_{0}, q_{1}}$. Let $\Psi$ be defined on $B$ by $\Psi\left(\left(q_{0}, q_{1}\right) \frown t\right)=$ $\Psi^{q_{0}, q_{1}}(t)$.

As $j+1 \notin \Theta_{\gamma}(\mathcal{Y})$ it is possible to use Lemma 3.1 to find $Y \in \mathcal{Y}$ such that $\Psi^{-1} Y \Subset_{\gamma}^{\mathfrak{M}} B$. In other words,

$$
\left\{\left(q_{1}, q_{0}\right) \in Q_{1} \times Q_{0} \mid\left(\Psi^{-1} Y\right)\left\langle\left(q_{1}, q_{0}\right)\right\rangle \Subset_{\gamma}^{\mathfrak{M}_{\left(q_{1}, q_{0}\right)}} B\left\langle\left(q_{1}, q_{0}\right)\right\rangle\right\} \Subset_{\gamma} \pi(B)=\bar{A} .
$$

Once again there are two cases to consider.

Case One. There is some $q_{1} \in A^{*}$ such that

$$
\mu_{0}\left(\left\{q_{0} \in Q_{0} \mid\left(q_{1}, q_{0}\right) \in \bar{A} \text { and }\left(\Psi^{-1} Y\right)\left\langle\left(q_{1}, q_{0}\right)\right\rangle \notin_{\gamma}^{\mathfrak{M}_{q_{1}, q_{0}}} B\left\langle\left(q_{1}, q_{0}\right)\right\rangle\right\}\right)<\gamma .
$$

Observe that $\mu_{0}\left(\bar{A}\left\langle q_{1}\right\rangle\right)>2 \epsilon(j)$ since $q_{1} \in A^{*}$. Furthermore, $B\left\langle\left(q_{1}, q_{0}\right)\right\rangle=B^{q_{1}, q_{0}}$ for any $q_{0} \in Q_{0}$. Moreover, $\Psi\left(\left(q_{1}, q_{0}\right)^{\frown} x\right)=\Psi^{q_{1}, q_{0}}(x)$ for any $x \in B^{q_{1}, q_{0}}$. Hence if

$$
E=\left\{q_{0} \in Q_{0} \mid\left(q_{1}, q_{0}\right) \in \bar{A} \text { and }\left(\Psi^{q_{1}, q_{0}}\right)^{-1} Y \Subset_{\gamma} B^{q_{1}, q_{0}}\right\},
$$

then $\mu_{0}(E) \geq 2 \epsilon(j)-\gamma>\epsilon(j)$ by the assumption on $\gamma$. Choose $x^{*} \in q_{1}$. Then if $p \in q_{0} \in E$ and $(x, p) \in A$ it cannot be the case that $Y \in\left\langle x^{*}, p, F\right\rangle_{j}$ since $\Psi^{q_{1}, q_{0}}$ was chosen to be a counterexample to $j \in \Theta_{\gamma}\left(\langle x, p, F\rangle_{j}\right)$. Therefore $\Lambda^{F(Y)}\left(p, x^{*}\right)>1 / j$ whenever $p \in \bigcup E$. Since $\sigma\left(\bigcup E \cap A\left\langle x^{*}\right\rangle\right)>(2 \epsilon(j)-\gamma) \zeta>\epsilon(j)$ it follows that $\lambda(F(Y))>J^{*}(j)$, contradicting that $F$ was chosen so that $\lambda(F(Y))<J^{*}(j)$.

Case Two. If $E$ is defined to be

$$
\left\{q_{1} \in A^{*} \mid\left(\forall q_{0} \in Q_{0}\right)\left(\Psi^{-1} Y\right)\left\langle\left(q_{1}, q_{0}\right)\right\rangle \notin_{\gamma}^{\mathfrak{M}_{q_{1}, q_{0}}} B\left\langle\left(q_{1}, q_{0}\right)\right\rangle \text { or }\left(q_{1}, q_{0}\right) \notin \bar{A}\right\},
$$

then $\lambda(E)<\gamma$.

Notice that $B\left\langle\left(q_{1}, q_{0}\right)\right\rangle \neq \emptyset$ if and only if $\left(q_{1}, q_{0}\right) \in \bar{A}$. Also,

$$
A^{*} \backslash E=\left\{q_{1} \in A^{*} \mid\left(\exists q_{0} \in Q_{0}\right)\left(\Psi^{q_{1}, q_{0}}\right)^{-1} Y \Subset_{\gamma}^{\mathfrak{M}_{q_{1}, q_{0}}} B^{q_{1}, q_{0}}\right\}
$$


and, moreover, $\mu_{1}\left(A^{*} \backslash E\right)>2 j^{q^{\prime}} K^{q^{\prime}} J^{*}(j)^{q^{\prime} / q}-\gamma>j^{q^{\prime}} K^{q^{\prime}} J^{*}(j)^{q^{\prime} / q}$. Furthermore, since the measure of $F(Y)$ is less than $J^{*}(j)$ it follows that

$$
\lambda\left(\left\{x \in[0,1]^{k} \mid(\exists p \in P) \Lambda^{F(Y)}(p, x) \geq 1 / j\right\}\right) \leq j^{q^{\prime}} K^{q^{\prime}} J^{*}(j)^{q^{\prime} / q}
$$

and so it is possible to choose $q_{1}^{*} \in A^{*}$ and $x^{*} \in q_{1}^{*}$ such that $\Lambda^{F(Y)}\left(p^{*}, x^{*}\right)<1 / j$ for all $p \in P$ and such that $\left(\Psi^{q_{1}^{*}, q_{0}^{*}}\right)^{-1} Y \Subset_{\gamma}^{\mathfrak{M}_{q_{1}, q_{0}}} B^{q_{1}^{*}}, q_{0}^{*}$ for some $q_{0}^{*} \in Q_{0}$. If $p^{*} \in q_{0}^{*} \cap A\left\langle x^{*}\right\rangle$, then $\Lambda^{F(Y)}\left(p^{*}, x^{*}\right)<1 / j$ implies that $Y \in\left\langle x^{*}, p^{*}, F\right\rangle_{j}$. However, the fact that $\left(\Psi^{q_{1}^{*}, q_{0}^{*}}\right)^{-1} Y \Subset_{\gamma}^{\mathfrak{M}_{q_{1}, q_{0}}} B^{q_{1}^{*}, q_{0}^{*}}$ contradicts the choice of $\Psi^{q_{1}^{*}, q_{0}^{*}}$ and $B^{q_{1}^{*}, q_{0}^{*}}$ and the fact that $\left(x^{*}, p^{*}\right) \in A$.

\section{The PIGEONHOLE PRINCIPLE}

The key step in justifying hypothesis 2.12 will be establishing a measuretheoretic pigeonhole principle. The description of the argument involves considerable notation, so an informal introduction to the reasoning behind this pigeonhole principle is likely to be helpful. Roughly speaking, it will be shown that for any $\gamma, \zeta>0$ and $j \in \mathbb{N}$ there is a sufficiently large integer $M$ such that for probability spaces $\left(P_{1}, \sigma_{1}\right)$ and $\left(P_{2}, \sigma_{2}\right)$ and any $\Psi: B \rightarrow M$ where $B \subseteq\left(P_{1} \times P_{2}\right)^{j}$ there is a $W \subseteq M$ such that $|W|<\zeta M$ and $\Psi^{-1} W \Subset_{\gamma} B$. For the purposes of this sketch it can be assumed that $B=\left(P_{1} \times P_{2}\right)^{j}$.

If $j=1$, then let $E_{x}=\left\{\Psi(x, y) \mid y \in P_{2}\right\}$ for each $x \in P_{1}$. Fix $k<\zeta M$ where $M$ has been chosen to be so large that $k$ can be assumed to have the following property: If $k$ elements of $M$ are painted white or black at random with black having probability $\zeta$, then the chances of having at least one element painted black are greater than $1-\gamma$. Given that $\left|E_{x}\right|>k$ for all $x$, choosing a random subset $W$ of $M$ by giving each element of $M$ a chance $\zeta$ of belonging to $W$ will yield a set $W$ such that $\Psi^{-1} W$ is likely to hit all but $\gamma$ of the $E_{x}$. In other words, $\Psi^{-1} W \Subset_{\gamma} P_{1} \times P_{2}$.

On the other hand, if there is even one $x$ such that $\left|E_{x}\right| \leq k$, then letting $W=E_{x}$ works. However, extending this strategy to even the case $j=2$ encounters some problems caused by the fact that $W$ is chosen probabilistically in one case, and according to a definite rule in the other. For example, if $j=2$, then for some $(x, y) \in P_{1} \times P_{2}$ the $W$ that works for the restriction of $\Psi$ to $\left(P_{1} \times P_{2}\right)^{2}\langle(x, y)\rangle$ may be chosen probabilistically for some $(x, y)$ while for others it might be $E_{z}$ for some $z$ such that $E_{x, y, z}$ is small, where $E_{x, y, z}=\left\{\Psi(x, y, z, w) \mid w \in P_{2}\right\}$. However, the probabilistic choice actually yields many $W$ that work; indeed, it will be argued that almost all $W$ will work.

But what happens if all of the $E_{x, y, z}$ are small? (This will be the assumption under which Sublemma 4.1 is proved.) If $M$ is sufficiently larger, then the argument for the case $j=1$ can be carried out with $k$ much smaller than $\zeta M$. Indeed, if $k$ is sufficiently small and there are sufficiently many $z$, choosing elements of $M$ at random will ensure that at least one of the $z$ will be lucky enough to have all the elements of $E_{x, y, z}$ chosen, provided the $\left\{E_{x, y, z}\right\}_{z \in P_{2}}$ are disjoint. But there is no reason to expect them to be disjoint. However, the following measure-theoretic version of the $\Delta$-system lemma, Lemma 4.1, shows that one can expect to have a large family of $z$ that form a $\Delta$-system with root $E_{x, y}$.

Lemma 4.1. Recall from $₫ 3$ that a probability space is said to be $(\delta, L)$-fine if and only if every set of measure greater than $\delta$ has cardinality at least $L$. If $L$ and $k$ are 
integers and $\tau$ a positive real, then there is an integer $\Xi(k, L, \tau) \geq k$ such that for any $\left(\tau / 2^{k}, L\right)$-fine probability space $(P, \sigma, \mathcal{M})$ and any measurable $Q: P \rightarrow[\mathbb{N}] \leq k$ there is $E \in[\mathbb{N}] \leq \Xi(k, L, \tau)$ such that for all $U \in \mathcal{M}$, if $\sigma(U) \geq \tau$ and $|U| \geq L$, then there is $X \in[U]^{L}$ such that the family $\{Q(x) \backslash E\}_{x \in X}$ is pairwise disjoint.

Now the $\left\{E_{x, y, z} \backslash E_{x, y}\right\}_{z}$ are pairwise disjoint for many $z$, guaranteeing that at least one of the $z$ will be lucky enough to have all the elements of $E_{x, y, z} \backslash E_{x, y}$ chosen. However, if this strategy is to be used to get a column witnessing that $\Psi^{-1} W \Subset_{\gamma}\left(P_{1} \times P_{2}\right)^{2}$ - in other words, to get $x \in P_{1}$ such that for most $y \in P_{2}$ there is $z$ such that all the elements of $E_{x, y, z} \backslash E_{x, y}$ are contained in $W$ - then the problem of getting $W$ to contain all of the remaining $E_{x, y}$ still exists.

Two alternatives have to be considered. In order to state these alternatives, use Lemma 4.1 to find, loosely speaking, a $\Delta$-system with root $E_{x}$ for the family $\left\{E_{x, y}\right\}_{y \in P_{2}}$ and a $\Delta$-system with root $E$ for the family $\left\{E_{x}\right\}_{x \in P_{1}}$. Either there is some $x \in P_{1}$ such that for most $y \in P_{2}$, for most $z \in P_{1},\left(E_{x} \backslash E\right) \cap E_{x, y, z} \neq \emptyset$ or there is not. If there is such an $x$, then let $W=E_{x} \backslash E$ and observe that $\left(\Psi^{-1} W\right)\langle(x, y)\rangle$ contains the graph of a function whose domain is almost all of $P_{1}$ for almost all $y \in P_{2}$. In other words, $\Psi^{-1} W \Subset_{\gamma}\left(P_{1} \times P_{2}\right)^{2}$ in this case.

On the other hand, if there is no such $x$, then for every $x \in P_{1}$ there are many $y \in P_{2}$ such that there are many $z \in P_{1}$ so that $\left(E_{x} \backslash E\right) \cap E_{x, y, z}=\emptyset$. The existence of many $y$ and $z$ allows Lemma 4.1 to be used to conclude that for any $x$, a randomly chosen $W$ is likely to have at least one $y_{x}$ such that there is at least one $z$ so that:

(1) $\left(E_{x} \backslash E\right) \cap E_{x, y_{x}, z}=\emptyset$,

(2) $E_{x, y_{x}, z} \backslash E_{x, y_{x}} \subseteq W$,

(3) $E_{x, y_{x}} \backslash E_{x} \subseteq W$

This means that one can expect $y_{x}$ to exist for all but $\gamma$ of the $x \in P_{1}$. In order for $\Psi^{-1} W \Subset_{\gamma}\left(P_{1} \times P_{2}\right)^{2}$ it suffices to establish that for each such $x$ there is $z \in P_{1}$ such that $E_{x, y_{x}, z} \subseteq W$. But it is immediate that

$$
\left(E_{x, y_{x}, z} \backslash E_{x, y_{x}}\right) \cup\left(E_{x, y_{x}} \backslash E_{x}\right) \subseteq W
$$

and, by choosing $M$ large enough, it can be guaranteed that $E$ is small enough that it can be included in $W$ as well. Hence, the only way that $E_{x, y_{x}, z}$ could fail to be a subset of $W$ is that $\left(E_{x} \backslash E\right) \cap E_{x, y_{x}, z} \nsubseteq W$. But, since $z$ can be chosen so that $\left(E_{x} \backslash E\right) \cap E_{x, y_{x}, z}=\emptyset$ this is no problem.

Arguments continuing this line of reasoning require a slight modification when $j>2$, but they will still yield Lemma 4.2. However, it should be apparent at this point that it is time to end the introductory sketch and to provide the necessary details and calculations. The first order of business is to establish the validity of Lemma 4.1

Proof of Lemma 4.1. Proceed by induction on $k$, the case $k=0$ being trivial since setting $\Xi(0, L, \tau)=0$ works for all $L$ and $\tau$. For $k \geq 1$ let $\Xi(k, L, \tau)$ be greater than $2 k L / \tau+\Xi(k-1, L, \tau / 2)$ and $k$. Given $Q$, let $\mathcal{A} \subseteq \mathcal{M}$ be a maximal pairwise disjoint family such that for each $A \in \mathcal{A}$,

- $\sigma(A) \geq \tau / 2$,

- there does not exist $X \in[A]^{L}$ such that $\{Q(x)\}_{x \in X}$ are pairwise disjoint. 
It follows that for each $A \in \mathcal{A}$ there is $E_{A} \in[\mathbb{N}]^{<k L}$ such that $Q(a) \cap E_{A} \neq \emptyset$ for each $a \in A$. Since $|\mathcal{A}| \leq 2 / \tau$ it follows that if $E^{1}$ is defined to be $\bigcup_{A \in \mathcal{A}} E_{A}$, then $\left|E^{1}\right| \leq 2 k L / \tau$.

Now define

$$
Q^{*}(p)= \begin{cases}Q(p) \backslash E^{1} & \text { if } p \in \bigcup \mathcal{A}, \\ \emptyset & \text { if } p \notin \cup \mathcal{A}\end{cases}
$$

and note that $Q^{*}: P \rightarrow[\mathbb{N}]^{\leq k-1}$ is measurable and the probability space $(P, \sigma)$ is $\left((\tau / 2) / 2^{k-1}, L\right)$-fine. By the induction hypothesis there is $E^{2} \in[\mathbb{N}]^{\Xi(k-1, L, \tau / 2)}$ such that for every $U \in \mathcal{M}$ such that $\sigma(U) \geq \tau / 2$ there is $X \in[U]^{L}$ such that the family $\left\{Q^{*}(x) \backslash E^{2}\right\}_{x \in X}$ is pairwise disjoint. Now let $E=E^{1} \cup E^{2}$ and note that $|E| \leq \Xi(k, L, \tau)$.

Moreover, if $U \in \mathcal{M}$ and $\sigma(U) \geq \tau$, then if $\sigma(U \backslash \cup \mathcal{A})>\tau / 2$ it follows from the maximality of $\mathcal{A}$ that there is some $X \in[U \backslash \cup \mathcal{A}]^{L}$ such that $\{Q(x)\}_{x \in X}$ are pairwise disjoint. Hence it may be assumed that $\sigma(U \cap(\bigcup \mathcal{A})) \geq \tau / 2$. Then there is $X \in[U \cap(\bigcup \mathcal{A})]^{L}$ such that $\left\{Q^{*}(x) \backslash E^{2}\right\}_{x \in X}$ are pairwise disjoint. In other words, $\{Q(x) \backslash E\}_{x \in X}$ are pairwise disjoint.

The next lemma is stated for finite probability spaces, but the proof shows that it also applies to Borel functions on Polish measure spaces all of whose projective subsets are measurable. Of course, for finite measure spaces or for finitely additive total measures on $\mathbb{N}$ this hypothesis is trivially satisfied but, if one assumes the measurability of all projective sets or the existence of real-valued measurable cardinals, then the argument of Lemma 4.2 has wider applicability. Whether these very strong hypotheses are actually required is not known.

Lemma 4.2. Suppose that $\xi \in(0,1), \gamma \in(0,1)$ and that $j$ is a non-zero integer. There is an integer $M(\xi, \gamma, j)$ and $\epsilon^{*}>0$ and $L^{*} \in \mathbb{N}$ such that for any two finite, $\left(\epsilon^{*}, L^{*}\right)$-fine, probability spaces $\left(P_{1}, \sigma_{1}\right)$ and $\left(P_{2}, \sigma_{2}\right)$ and any $B \subseteq\left(P_{1} \times P_{2}\right)^{j}$ and any function $\Psi: B \rightarrow M(\xi, \gamma, j)$ there is $W \in \mathcal{P}_{\xi}(M(\xi, \gamma, j))$ such that $\Psi^{-1} W \Subset_{\gamma}$ $B$.

Proof. If $\xi, \gamma$ and $j$ have been given, define $\bar{\gamma}=\gamma / 2$ and let $\bar{\xi}=\xi /(2(j+1))$. Define $\beta(0, \delta)=1-\delta$ and then inductively define

$$
\beta(n+1, \delta)=1-\frac{\max (1-\beta(n, \delta)(1-\delta)+\delta, 1-\beta(n, \delta), \delta)}{\bar{\gamma}}
$$

for $\delta>0$. It follows by induction that $\lim _{\delta \rightarrow 0} \beta(n, \delta)=1$ for each $n$, so choose $\bar{\delta} \in(0, \bar{\gamma})$ so small that $\beta(j, \bar{\delta})>1 / 2$. Let $\beta(i)=\beta(i, \bar{\delta})$. Observe that $\bar{\gamma} \leq 1 / 2$ implies that $\beta(i) \geq \beta(i+1)$ for all $i$.

Begin by choosing $k_{1} \in \mathbb{N}$ so large that

$$
(1-\bar{\xi})^{k_{1}}<\bar{\delta}
$$

and, given $k_{n}$, choose $L_{n}$ so large that

$$
\left(1-\bar{\xi}^{k_{n}}\right)^{L_{n}}<\bar{\delta}
$$

and, given $L_{n}$, let

$$
k_{n+1}=\Xi\left(k_{n}, L_{n}, \bar{\delta}\right)
$$


where $\Xi$ is the function described in Lemma 4.1. Since the function $\Xi$ satisfies that $\Xi(k, L, \tau) \geq k$ the sequence $\left\{k_{n}\right\}_{n=1}^{2 j+1}$ is monotone. Let $\epsilon^{*}=\bar{\delta} / 2^{k_{2 j+1}}$ and $L^{*}=L_{2 j+1}$.

Then let $M=M(\xi, \gamma, j) \in \mathbb{N}$ be sufficiently large that

$$
\frac{k_{2 j+1}}{M}<\bar{\xi}
$$

and, furthermore, such that the probability that $\sum_{i=1}^{M} X_{i}<2 \bar{\xi} M$ is greater than $2^{-1 / j}$, where $\left\{X_{i}\right\}_{i=1}^{M}$ are independent $\{0,1\}$-valued random variables with mean $\bar{\xi}$. In order to avoid dealing with probabilities, however, let $\bar{\mu}$ be the probability measure on $\{0,1\}$ defined by $\bar{\mu}(\{1\})=\bar{\xi}$ and $\bar{\mu}(\{0\})=1-\bar{\xi}$ and let $\mu=\bar{\mu}^{M}$ be the $M$-fold product of $\bar{\mu}$ on $2^{M}$. Elements of $2^{M}$ will be identified with subsets of $M$ by their supports; in other words, $\mu$ will be thought of as a measure on $\mathcal{P}(M)$ and hence the probability inequality following inequality 4.5 is equivalent to

$$
\mu(\{W \subseteq M|| W \mid<2 \bar{\xi} M\})>\frac{1}{2^{1 / j}} .
$$

In preparation for the proof some conventions for denoting coordinates will be introduced. If $z \in\left(P_{1} \times P_{2}\right)^{m}$, then

$$
z=\left(\left(z_{1}(1), z_{2}(1)\right),\left(z_{1}(2), z_{2}(2)\right), \ldots,\left(z_{1}(m), z_{2}(m)\right)\right)
$$

while if $z \in\left(P_{1} \times P_{2}\right)^{m} \times P_{1}$, then

$$
z=\left(\left(z_{1}(1), z_{2}(1)\right),\left(z_{1}(2), z_{2}(2)\right), \ldots,\left(z_{1}(m), z_{2}(m)\right), z(m+1)\right) .
$$

For $z \in\left(P_{1} \times P_{2}\right)^{j}$ and, for any $\ell \leq j$, let

$$
\begin{gathered}
z_{1}^{*}(\ell)=\left(\left(z_{1}(1), z_{2}(1)\right),\left(z_{1}(2), z_{2}(2)\right), \ldots,\left(z_{1}(\ell-1), z_{2}(\ell-1)\right), z_{1}(\ell)\right), \\
z^{*}(\ell)=\left(\left(z_{1}(1), z_{2}(1)\right),\left(z_{1}(2), z_{2}(2)\right), \ldots,\left(z_{1}(\ell), z_{2}(\ell)\right)\right)
\end{gathered}
$$

and $z^{*}(0)$ will be defined to be $\emptyset$. If $z \in\left(P_{1} \times P_{2}\right)^{m}$ and $x \in P_{1}$, then $z^{-} x$ will denote

$$
\left(\left(z_{1}(1), z_{2}(1)\right),\left(z_{1}(2), z_{2}(2)\right), \ldots,\left(z_{1}(m), z_{2}(m)\right), x\right)
$$

with the convention that $\emptyset \subset x=x$ in the case $m=0$. If $z \in\left(P_{1} \times P_{2}\right)^{m} \times P_{1}$ and $x \in P_{2}$, then $z^{\frown} x$ will denote

$$
\left(\left(z_{1}(1), z_{2}(1)\right),\left(z_{1}(2), z_{2}(2)\right), \ldots,\left(z_{1}(m), z_{2}(m)\right),(z(m+1), x)\right) .
$$

Now suppose that $\left(P_{1}, \sigma_{1}\right)$ and $\left(P_{2}, \sigma_{2}\right)$ are finite, $\left(\epsilon^{*}, L^{*}\right)$-fine, probability spaces and that $B \subseteq\left(P_{1} \times P_{2}\right)^{j}$ and $\Psi: B \rightarrow M$. For $x \in\left(P_{1} \times P_{2}\right)^{j-1} \times P_{1}$ define $E_{x}=\{\Psi(x \frown y) \mid y \in B\langle x\rangle\}$. Before considering the general case, assume that $\left|E_{x}\right| \leq k_{1}$ for each $x \in\left(P_{1} \times P_{2}\right)^{j-1} \times P_{1}$. It will first be shown that in this case the following sublemma holds. Most of the argument to follow will be devoted to this special case; the general result will then follow with only a little more effort.

Sublemma 4.1. There is $W \subseteq \mathcal{P}_{(2 j+1) \bar{\xi}}(M)$ such that $\Psi^{-1} W \Subset_{\bar{\gamma}} B$.

Proof. If $j=1$, then choosing any $x \in P_{1}$ such that $B\langle x\rangle \neq \emptyset$ and letting $W=E_{x}$ will satisfy that $\Psi^{-1} W \Subset_{\gamma} B$ and $|W| \leq k_{1} \leq k_{3} \leq \bar{\xi} M$. Therefore assume that $j \geq 2$. 
Now suppose that $1 \leq \ell \leq j$ and that $E_{x} \in[M] \leq k_{2 \ell-1}$ has been defined for each $x \in\left(P_{1} \times P_{2}\right)^{j-\ell} \times P_{1}$. For $x \in\left(P_{1} \times P_{2}\right)^{j-\ell}$ let $E_{x} \subseteq M$ be such that

$$
\left|E_{x}\right| \leq \Xi\left(k_{2 \ell-1}, L_{2 \ell-1}, \bar{\delta}\right)=k_{2 \ell},
$$

$\left(\forall U \subseteq P_{1}\right)$ if $\sigma_{1}(U) \geq \bar{\delta}$, then $\left(\exists Z \in[U]^{L_{2 \ell-1}}\right)\left\{E_{x \frown z} \backslash E_{x}\right\}_{z \in Z}$ is pairwise disjoint using the convention that $\left(P_{1} \times P_{2}\right)^{0}=\{\emptyset\}$ and $\left(P_{1} \times P_{2}\right)^{0} \times P_{1}=P_{1}$ in the case that $j=\ell$. On the other hand, suppose that $1 \leq \ell<j$ and that $E_{x} \in[M] \leq k_{2 \ell}$ has been defined for each $x \in\left(P_{1} \times P_{2}\right)^{j-\ell}$. For $x \in\left(P_{1} \times P_{2}\right)^{j-\ell-1} \times P_{1}$ let $E_{x} \subseteq M$ be such that

$\left(\forall U \subseteq P_{2}\right)$ if $\sigma_{2}(U) \geq \bar{\delta}$, then $\left(\exists Z \in[U]^{L_{2 \ell}}\right)\left\{E_{x \frown z} \backslash E_{x}\right\}_{z \in Z}$ is pairwise disjoint.

Now begin by defining

$$
C_{1}=\left\{z \in\left(P_{1} \times P_{2}\right)^{j-1} \times P_{1} \mid B\langle z\rangle \neq \emptyset \text { and } \bigcup_{\ell=1}^{j-1}\left(E_{z_{1}^{*}(\ell)} \backslash E_{z^{*}(\ell-1)}\right) \cap E_{z}=\emptyset\right\}
$$

and, given that $C_{\ell} \subseteq\left(P_{1} \times P_{2}\right)^{j-\ell} \times P_{1}$ has been defined, for each $z \in\left(P_{1} \times P_{2}\right)^{j-\ell}$ let $\Theta(z)=C_{\ell}\langle z\rangle$. For $z \in\left(P_{1} \times P_{2}\right)^{j-(\ell+1)} \times P_{1}$ let

$$
\Omega(z)=\left\{y \in P_{2} \mid \sigma_{1}(\Theta(z \frown y))>\bar{\delta}\right\}
$$

if $\ell=1$ while if $1<\ell<j$, then let

$$
\Omega(z)=\left\{y \in P_{2} \mid \sigma_{1}(\Theta(z \frown y))<\bar{\delta}\right\}
$$

noting the crucial difference in the two definitions. Then let

$$
C_{\ell+1}=\left\{z \in\left(P_{1} \times P_{2}\right)^{j-(\ell+1)} \times P_{1} \mid B\langle z\rangle \neq \emptyset \text { and } \sigma_{2}(\Omega(z))<\bar{\delta}\right\} .
$$

There are now two cases to consider.

Case One. $C_{j} \neq \emptyset$.

Define $H_{\ell}$ by induction on $\ell \geq 2$ to be an $(\ell-2)$-ary function on $\mathcal{P}(M)$. If $\ell=2$, then let $H_{2}=C_{2}$ and if $\ell \geq 3$, define $H_{\ell}\left(W_{1}, W_{2}, \ldots, W_{\ell-2}\right)$ to be the set of all $x \in C_{\ell}$ such that

$$
\sigma_{2}\left(H_{\ell}^{*}\left(x, W_{1}, W_{2}, \ldots, W_{\ell-2}\right)\right)<\bar{\gamma},
$$

where $H_{\ell}^{*}\left(x, W_{1}, W_{2}, \ldots, W_{\ell-2}\right)$ is defined to be

$\left\{y \in P_{2} \mid\left(\forall z \in P_{1}\right) E_{x \frown y \frown z} \backslash E_{x \frown y} \nsubseteq W_{1}\right.$ or $\left.x \frown y \frown z \notin H_{\ell-1}\left(W_{2}, W_{3}, \ldots, W_{\ell-2}\right)\right\}$.

Claim 1. If $\ell \geq 3$ and $x \in C_{\ell}$, then $\mu^{\ell-2}(D(x)) \geq \beta(\ell)$, where $D(x)=$ $\left\{\vec{W} \in \mathcal{P}(M)^{\ell-2} \mid x \in H_{\ell}(\vec{W})\right\}$.

Proof. The argument will proceed by induction on $\ell$. In preparation for the main argument, however, observe that since $\ell \geq 3$, then for any $y \in P_{2} \backslash \Omega(x)$ it must be the case that $\sigma_{1}\left(\Theta\left(x^{\frown} y\right)\right) \geq \bar{\delta}$. Hence there is $Z(y) \in\left[\Theta\left(x^{\frown} y\right)\right]^{L_{2 \ell-3}}$ such that $\left\{E_{x \frown y \frown z} \backslash E_{x \frown y}\right\}_{z \in Z(y)}$ is a pairwise disjoint family. Therefore

$$
\mu\left(D_{0}(x \frown y)\right)=1-\prod_{z \in Z(y)}\left(1-\bar{\xi}^{\left|E_{x} \frown y \frown z \backslash E_{x} \frown y\right|}\right) \geq 1-\left(1-\bar{\xi}^{k_{2 \ell-3}}\right)^{L_{2 \ell-3}}>1-\bar{\delta},
$$




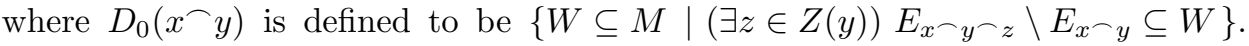
Let $\left\{D_{1}\left(x^{\frown} y\ulcorner z)\right\}_{z \in Z(y)}\right.$ be a partition of $D_{0}\left(x^{\frown} y\right)$ such that if $W \in D_{1}\left(x^{\frown} y\ulcorner z)\right.$, then $E_{x \frown y \frown z} \backslash E_{x \frown y} \subseteq W$. Moreover, since $Z(y) \subseteq \Theta(x \frown y)$ it follows that $x \frown y \frown z \in C_{\ell-1}$ if $z \in Z(y)$.

The initial case of the induction is $\ell=3$. In this case, if $y \in P_{2} \backslash \Omega(x)$, then

$$
\begin{aligned}
\geq \mu\left(\left\{W \mid(\exists z \in Z(y)) E_{x \frown y \frown z} \backslash E_{x \frown y} \subseteq W\right\}\right) \geq \mu\left(\bigcup_{z \in Z(y)} D_{1}(x \frown y \frown z)\right) \\
=\mu\left(D_{0}\left(x^{-} y\right)\right)>1-\bar{\delta} \geq \beta(2)(1-\bar{\delta})
\end{aligned}
$$

by equality (4.1). On the other hand, if $\ell>3$ and $y \in P_{2} \backslash \Omega(x)$, then the induction hypothesis implies that $\mu^{\ell-3}(D(x \frown y \frown z)) \geq \beta(\ell-1)$ for any $z \in Z(y)$ and therefore if $\ell>3$, then

$$
\begin{aligned}
& \mu^{\ell-2}\left(\left\{\left(W_{1}, W_{2}, \ldots, W_{\ell-2}\right) \mid(\exists z) E_{x \frown y \frown z} \backslash E_{x \frown y} \subseteq W_{1}\right.\right. \text { and } \\
& \left.\left.x \frown y \frown z \in H_{\ell-1}\left(W_{2}, W_{3}, \ldots, W_{\ell-2}\right)\right\}\right) \\
& \geq \mu^{\ell-2}\left(\bigcup_{z \in Z(y)} D_{1}\left(x^{\frown} y \frown z\right) \times D\left(x^{\frown} y \frown z\right)\right) \geq \sum_{z \in Z(y)} \mu\left(D_{1}\left(x^{\frown} y \frown z\right)\right) \beta(\ell-1) \\
& =\mu\left(D_{0}\left(x^{\frown} y\right)\right) \beta(\ell-1)>(1-\bar{\delta}) \beta(\ell-1) .
\end{aligned}
$$

In either case, either inequality (4.12) or inequality (4.13), it follows that for any $y \in P_{2} \backslash \Omega(x)$,

$$
\mu^{\ell-2}\left(\left\{\vec{W} \mid y \in H_{\ell}^{*}(x, \vec{W})\right\}\right)<1-(1-\bar{\delta}) \beta(\ell-1)
$$

and so

$$
\int_{P_{2} \backslash \Omega(x)} \mu^{\ell-2}\left(\left\{\vec{W} \mid y \in H_{\ell}^{*}(x, \vec{W})\right\}\right) d \sigma_{2}(y)<1-(1-\bar{\delta}) \beta(\ell-1) .
$$

Hence

$$
\int_{P_{2}} \mu^{\ell-2}\left(\left\{\vec{W} \mid y \in H_{\ell}^{*}(x, \vec{W})\right\}\right) d \sigma_{2}(y)<1-(1-\bar{\delta}) \beta(\ell-1)+\bar{\delta}
$$

and therefore, by Fubini's Theorem,

$$
\int_{\mathcal{P}(M)^{\ell-2}} \sigma_{2}\left(H_{\ell}^{*}(x, \vec{W})\right) d \mu^{\ell-2}(\vec{W})<1-\beta(\ell-1)(1-\bar{\delta})+\bar{\delta} .
$$

Therefore, using inequality (4.11) and Markov's Inequality,

$$
\begin{aligned}
\mu^{\ell-2} & \left(\left\{\vec{W} \in \mathcal{P}(M)^{\ell-2} \mid x \in H_{\ell}(\vec{W})\right\}\right) \\
& =\mu^{\ell-2}\left(\left\{\vec{W} \in \mathcal{P}(M)^{\ell-2} \mid \sigma_{2}\left(H_{\ell}^{*}(x, \vec{W})\right)<\bar{\gamma}\right\}\right) \\
& \geq 1-\frac{1-\beta(\ell-1)(1-\bar{\delta})+\bar{\delta}}{\bar{\gamma}}
\end{aligned}
$$

and comparing this with the definition of $\beta(\ell)$ establishes that the claim is proved. 
Claim 2. Let $\ell \geq 2$. If $x \in H_{\ell}\left(W_{2}, W_{3}, \ldots, W_{\ell-1}\right)$ and $W_{1} \supseteq E_{x} \backslash E_{x^{*}(j-\ell)}$, then

$$
\Psi^{-1}\left(\left(\bigcup_{i=1}^{j-\ell} E_{x_{1}^{*}(i)} \backslash E_{x^{*}(i-1)}\right) \cup \bigcup_{i=1}^{\ell-1} W_{i}\right)\left\langle x^{*}(j-\ell)\right\rangle \Subset_{\bar{\gamma}} B\left\langle x^{*}(j-\ell)\right\rangle
$$

using the convention that $\bigcup_{i=1}^{0} E_{x_{1}^{*}(i)} \backslash E_{x^{*}(i-1)}=\emptyset$ in the case that $\ell=j$.

Proof. This is established by induction on $\ell$. If $\ell=2$, then define $E^{\prime}=\bigcup_{i=1}^{j-1} E_{x_{1}^{*}(i)} \backslash$ $E_{x^{*}(i-1)}$ and note that

$$
\left(\bigcup_{i=1}^{j-2} E_{x_{1}^{*}(i)} \backslash E_{x^{*}(i-1)}\right) \cup W_{1} \supseteq E^{\prime} .
$$

Since $x \in C_{2}$ it follows that $\sigma_{2}(\Omega(x))<\bar{\delta}$ and $B\langle x\rangle \neq \emptyset$. Furthermore, if $y \in \Omega(x)$, then $\sigma_{1}(\Theta(x \frown y))>\bar{\delta}$ because of the special definition in the case $x \in\left(P_{1} \times P_{2}\right)^{j-2} \times$ $P_{1}$. In other words, noting that $x \frown y \in\left(P_{1} \times P_{2}\right)^{j-1}$ and paying attention to the definition of $C_{1}$,

$$
\sigma_{2}\left(\left\{y \in P_{2} \mid \sigma_{1}\left(\left\{z \in P_{1} \mid E^{\prime} \cap E_{x \frown y \frown z}=\emptyset\right\}\right)>\bar{\delta}\right\}\right)<\bar{\delta}
$$

and so, recalling that $E_{x \frown y \frown z}$ is the image of $\{x \frown y \frown z\} \times B\langle x \frown y \frown z\rangle$ under $\Psi$,

$$
\sigma_{2}\left(\left\{y \in P_{2} \mid \sigma_{1}\left(\left\{z \in P_{1} \mid \Psi^{-1} E^{\prime}\left\langle x^{\frown} y^{\frown} z\right\rangle=\emptyset\right\}\right)>\bar{\delta}\right\}\right)<\bar{\delta}
$$

and so $x_{1}^{*}(j-1)$ witnesses that $\Psi^{-1}\left(E^{\prime}\right)\left\langle x^{*}(j-2)\right\rangle \Subset_{\bar{\delta}} B\left\langle x^{*}(j-2)\right\rangle$ according to alternative (3.1) of Definition 3.1. Because $\bar{\delta}<\bar{\gamma}$ and inclusion (4.14) holds, the result now follows.

For the induction step suppose the claim has been proved for $\ell-1$ and that $W_{1} \supseteq E_{x} \backslash E_{x^{*}(j-\ell)}$ and $x \in H_{\ell}\left(W_{2}, W_{3}, \ldots, W_{\ell-1}\right)$. From the induction hypothesis it follows that if $E_{x \frown y \frown z} \backslash E_{x \frown y} \subseteq W_{2}$ and $x \frown y \frown z \in H_{\ell-1}\left(W_{3}, W_{4}, \ldots, W_{\ell-1}\right)$, then

$$
\Psi^{-1}\left(\left(\bigcup_{i=1}^{j-(\ell-1)} E_{(x \frown y \frown z)_{1}^{*}(i)} \backslash E_{(x-y \frown z)^{*}(i-1)}\right) \cup \bigcup_{i=2}^{\ell-1} W_{i}\right)\langle x \frown y\rangle \Subset_{\bar{\gamma}} B\langle x \frown y\rangle .
$$

Note that $(x \frown y \frown z)_{1}^{*}(j-(\ell-1))=x$ and, since $W_{1} \supseteq E_{x} \backslash E_{x^{*}(j-\ell)}$, it follows that

$$
\left(\bigcup_{i=1}^{j-(\ell-1)} E_{(x-y-z)_{1}^{*}(i)} \backslash E_{(x-y-z)^{*}(i-1)}\right) \cup \bigcup_{i=2}^{\ell-1} W_{i} \subseteq\left(\bigcup_{i=1}^{j-\ell} E_{x_{1}^{*}(i)} \backslash E_{x^{*}(i-1)}\right) \cup \bigcup_{i=1}^{\ell-1} W_{i} .
$$

Hence,

$$
\begin{array}{r}
\sigma_{2}\left(\left\{y \in P_{2} \mid \Psi^{-1}\left(\left(\bigcup_{i=1}^{j-\ell} E_{x_{1}^{*}(i)} \backslash E_{x^{*}(i-1)}\right) \cup \bigcup_{i=1}^{\ell-1} W_{i}\right)\langle x \frown y\rangle \notin_{\bar{\gamma}} B\langle x \frown y\rangle\right\}\right) \\
\leq \sigma_{2}\left(H_{\ell}^{*}\left(x, W_{2}, W_{3}, \ldots, W_{\ell-1}\right)\right)<\bar{\gamma} .
\end{array}
$$

But note that $x \frown y$ in inequality (4.15) is the same as $x^{*}(j-\ell) \frown x_{1}(j-(\ell-1)) \frown y$ and so $x_{1}(j-(\ell-1))$ is a witness to the fact that

$$
\Psi^{-1}\left(\left(\bigcup_{i=1}^{j-\ell} E_{x_{1}^{*}(i)} \backslash E_{x^{*}(i-1)}\right) \cup \bigcup_{i=1}^{\ell-1} W_{i}\right)\left\langle x^{*}(j-\ell)\right\rangle \Subset_{\bar{\gamma}} B\left\langle x^{*}(j-\ell)\right\rangle
$$


satisfies alternative (3.1) of Definition 3.1. Moreover, $x \in H_{\ell}\left(W_{2}, W_{3}, \ldots, W_{\ell-1}\right) \subseteq$ $C_{\ell}$ implies $B\langle x\rangle \neq \emptyset$. Hence $B\left\langle x^{*}(j-\ell\rangle\left\langle x_{1}(j-(\ell-1))\right\rangle \neq \emptyset\right.$, as required.

Now choose $x \in C_{j}$. If $j=2$, then $x \in H_{2}$ and so Claim 2 and inequality (4.5) can be directly applied to find $W$ such that $E_{x} \subseteq W \subseteq M$ and $|W| \leq k_{3}<\bar{\xi} M$. If $j \geq 3$, then from Claim 1 and the fact that $\beta(j)>1 / 2$ and inequality (4.6) it follows that there are $\left\{W_{i}\right\}_{i=2}^{j-1}$ such that $\left|W_{i}\right|<2 \bar{\xi} M$ for each $i$ and such that $x \in H_{j}\left(W_{2}, W_{3}, \ldots, W_{j-1}\right)$. Let $W_{1}=E_{x}$ and define $W=\bigcup_{i=1}^{j-1} W_{i}$. It follows from inequality (4.5) that

$$
|W|<2(j-2) \bar{\xi} M+k_{2 j+1}<(2 j+1) \bar{\xi} M
$$

and, moreover, the hypotheses of Claim 2 are satisfied by $W_{1}, W_{2}, \ldots, W_{\ell-1}$ and $x$. Therefore

$$
\Psi^{-1}\left(\left(\bigcup_{i=1}^{j-j} E_{x_{1}^{*}(i)} \backslash E_{x^{*}(i-1)}\right) \cup W\right)\left\langle x^{*}(j-j)\right\rangle \Subset_{\bar{\gamma}} B\left\langle x^{*}(j-j)\right\rangle
$$

and so $\Psi^{-1} W \Subset_{\bar{\gamma}} B$.

Case Two. $C_{j}=\emptyset$.

The general scheme of the proof is the same as in the first case. However, the lack of symmetry between $P_{1}$ and $P_{2}$ requires some slight modifications of the argument. Define an $\ell$-ary function $H_{\ell}$ on $\mathcal{P}(M)$ by induction on $\ell$. If $\ell=1$, then

$$
H_{1}(W)=\left\{x \in\left(P_{1} \times P_{2}\right)^{j-1} \mid B\langle x\rangle=\emptyset \text { or }(\exists z \in \Theta(x)) E_{x \frown z} \backslash E_{x} \subseteq W\right\}
$$

and if $\ell \geq 2$, define $H_{\ell}\left(W_{1}, W_{2}, \ldots, W_{\ell}\right)$ to be the set of all $x \in\left(P_{1} \times P_{2}\right)^{j-\ell}$ such that

$$
\sigma_{1}\left(H_{\ell}^{*}\left(x, W_{1}, W_{2}, \ldots, W_{\ell}\right)\right)<\bar{\gamma}
$$

where $H_{\ell}^{*}\left(x, W_{1}, W_{2}, \ldots, W_{\ell}\right)$ is defined to be

$$
\left\{y \in P_{1} \mid\left(\forall z \in P_{2}\right) E_{x \frown y \frown z} \backslash E_{x \frown y} \not \subset W_{1} \text { or } x \frown y \frown z \notin H_{\ell-1}\left(W_{2}, \ldots, W_{\ell}\right)\right\} .
$$

Claim 3. If $x \in\left(P_{1} \times P_{2}\right)^{j-\ell}$ and either

- $B\langle x\rangle=\emptyset$,

- $\ell=1$ and $\sigma_{1}(\Theta(x)) \geq \bar{\delta}$,

- or $\ell>1$ and $\sigma_{1}(\Theta(x))<\bar{\delta}$,

then $\mu^{\ell}(D(x))>\beta(\ell)$, where $D(x)=\left\{\vec{W} \in \mathcal{P}(M)^{\ell} \mid x \in H_{\ell}(\vec{W})\right\}$.

Proof. Begin by considering the case that $\ell=1$ in which there are two possibilities. The first is that $B\langle x\rangle=\emptyset$ and in this case it suffices to observe that $D(x)=\mathcal{P}(M)$. In the second case $\sigma_{1}(\Theta(x)) \geq \bar{\delta}$ and so there is then $Z \in[\Theta(x)]^{L_{1}}$ such that $\left\{E_{x \frown z} \backslash E_{x}\right\}_{z \in Z}$ is a pairwise disjoint family and hence

$$
\mu\left(\left\{W \subseteq M \mid(\forall z \in Z) E_{x \frown z} \backslash E_{x} \nsubseteq W\right\}\right)<\bar{\delta}
$$

by inequality (4.3). Hence $\mu\left(\left\{W \subseteq M \mid x \in H_{1}(W)\right\}\right)>1-\bar{\delta} \geq \beta(1)$.

On the other hand, if $\ell>1$ and $\sigma_{1}(\Theta(x))<\bar{\delta}$, then note that if $y \in P_{1} \backslash$ $\Theta(x)$, then $x^{\frown} y \notin C_{\ell}$ and so there are again two possibilities: either $B\langle x \frown y\rangle=\emptyset$ or $\sigma_{2}\left(\Omega\left(x^{\frown} y\right)\right) \geq \bar{\delta}$. In the second case there is $Z \in\left[\Omega\left(x^{\frown} y\right)\right]^{L_{2(\ell-1)}}$ such that $\left\{E_{x \frown y \frown z} \backslash E_{x \frown y}\right\}_{z \in Z}$ is a pairwise disjoint family. Therefore

$$
\mu\left(D_{0}(x \frown y)\right) \geq 1-\left(1-\bar{\xi}^{k_{2(\ell-1)}}\right)^{L_{2(\ell-1)}}>1-\bar{\delta},
$$




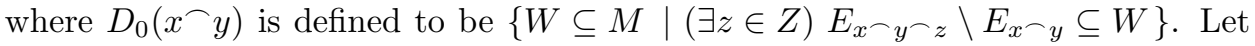
$\left\{D_{1}(x \frown y \frown z)\right\}_{z \in Z}$ be a partition of $D_{0}(x \frown y)$ such that if $W \in D_{1}\left(x^{\frown} y\ulcorner z)\right.$, then

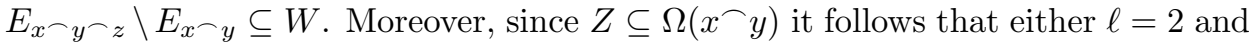
$\sigma_{1}(\Theta(x \frown y \frown z)) \geq \bar{\delta}$ for all $z \in Z$ or, $\ell>2$ and $\sigma_{1}(\Theta(x \frown y \frown z))<\bar{\delta}$ for all $z \in Z$. In either case the hypothesis of the claim holds for $x \frown y \frown z \in\left(P_{1} \times P_{2}\right)^{j-(\ell-1)}$ and so the induction hypothesis implies that $\mu^{\ell-1}(D(x \frown y \frown z))>\beta(\ell-1)$ for any $z \in Z$. Therefore

$$
\begin{aligned}
& \mu^{\ell}\left(\left\{\left(W_{1}, W_{2}, \ldots, W_{\ell}\right) \mid(\exists z) E_{x \frown y \frown z \backslash E_{x \frown y} \subseteq W_{1} \text { and }}\right.\right. \\
& \left.\left.x \frown y \frown z \in H_{\ell-1}\left(W_{2}, W_{3}, \ldots, W_{\ell}\right)\right\}\right) \\
& \geq \mu^{\ell}\left(\bigcup_{z \in Z} D_{1}\left(x^{\frown} y \frown z\right) \times D(x \frown y \frown z)\right) \geq \sum_{z \in Z} \mu\left(D_{1}\left(x^{\frown} y \frown z\right)\right) \beta(\ell-1) \\
& \quad=\mu\left(D_{0}(x \frown y)\right) \beta(\ell-1)>(1-\bar{\delta}) \beta(\ell-1)
\end{aligned}
$$

for all $y \in P_{1} \backslash \Theta(x)$.

If $B\langle x \frown y\rangle=\emptyset$, then $E_{z}=\emptyset$ for any $z \supseteq x^{\frown} y$. It then follows from the induction hypothesis that

$$
\begin{aligned}
& \mu^{\ell}\left(\left\{\left(W_{1}, W_{2}, \ldots, W_{\ell}\right) \mid(\exists z) E_{x \frown y \frown z \backslash E_{x \frown y} \subseteq W_{1} \text { and }}\right.\right. \\
& \left.\left.x \frown y \frown z \in H_{\ell-1}\left(W_{2}, W_{3}, \ldots, W_{\ell}\right)\right\}\right) \\
& \quad \geq \mu^{\ell}(\mathcal{P}(M) \times D(x \frown y \frown z)) \geq \beta(\ell-1) .
\end{aligned}
$$

The rest of the proof of Claim 3 is the same as the proof of Claim 1 with the probability space $\left(P_{1}, \sigma_{1}\right)$ playing the role of $\left(P_{2}, \sigma_{2}\right)$ and $\ell$ in place of $\ell-2$.

Claim 4. If $x \in H_{\ell}\left(W_{1}, W_{2}, \ldots, W_{\ell}\right)$, then

$$
\Psi^{-1}\left(E_{\emptyset} \cup \bigcup_{i=1}^{j-\ell}\left(E_{x^{*}(i)} \backslash E_{x_{1}^{*}(i)}\right) \cup \bigcup_{i=1}^{\ell} W_{i}\right)\langle x\rangle \Subset_{\bar{\gamma}} B\langle x\rangle .
$$

Proof. This is also proved by induction on $\ell$. If $x \in H_{1}(W)$ and $B\langle x\rangle=\emptyset$ the result is trivial. Otherwise there is some $z \in \Theta(x)$ such that $E_{x-z} \backslash E_{x} \subseteq W$. Since $z \in \Theta(x)$ it follows that $x \frown z \in C_{1}$ and so

$$
\bigcup_{i=1}^{j-1}\left(E_{x_{1}^{*}(i)} \backslash E_{x^{*}(i-1)}\right) \cap E_{x \frown z}=\emptyset
$$

and since

$$
E_{x \frown z} \subseteq\left(E_{x \frown z} \backslash E_{x}\right) \cup E_{\emptyset} \cup\left(\bigcup_{i=1}^{j-1}\left(E_{x^{*}(i)} \backslash E_{x_{1}^{*}(i)}\right) \cup\left(E_{x_{1}^{*}(i)} \backslash E_{x^{*}(i-1)}\right)\right),
$$

it follows from identity (4.20) that

$$
E_{x \frown z} \subseteq\left(E_{x \frown z} \backslash E_{x}\right) \cup E_{\emptyset} \cup \bigcup_{i=1}^{j-1}\left(E_{x^{*}(i)} \backslash E_{x_{1}^{*}(i)}\right) \subseteq W \cup E_{\emptyset} \cup \bigcup_{i=1}^{j-1}\left(E_{x^{*}(i)} \backslash E_{x_{1}^{*}(i)}\right)
$$

or, in other words,

$$
\emptyset \neq B\langle x \frown z\rangle \subseteq \Psi^{-1}\left(W \cup E_{\emptyset} \cup \bigcup_{i=1}^{j-1}\left(E_{x^{*}(i)} \backslash E_{x_{1}^{*}(i)}\right)\right)\langle x \frown z\rangle .
$$


Therefore $z$ is a witness to the fact that

$$
\Psi^{-1}\left(E_{\emptyset} \cup \bigcup_{i=1}^{j-1}\left(E_{x^{*}(i)} \backslash E_{x_{1}^{*}(i)}\right) \cup W\right)\langle x\rangle \Subset_{\bar{\gamma}} B\langle x\rangle .
$$

Now assume that $\ell>1$ and $x \in H_{\ell}\left(W_{1}, W_{2}, \ldots, W_{\ell}\right)$. In other words, since $\ell>1$ it must be the case that inequality (4.16) holds. From the induction hypothesis and Definition 4.17 it follows that if $y \in P_{1} \backslash H_{\ell}^{*}\left(x, W_{1}, W_{2}, \ldots, W_{\ell}\right)$, then there exists $z \in P_{2}$ such that $E_{x \frown y \frown z} \backslash E_{x \frown y} \subseteq W_{1}$ and

$$
\begin{array}{r}
\Psi^{-1}\left(E_{\emptyset} \cup\left(E_{x \frown y \frown z} \backslash E_{x \frown y}\right) \cup \bigcup_{i=1}^{j-\ell}\left(E_{x^{*}(i)} \backslash E_{x_{1}^{*}(i)}\right) \cup \bigcup_{i=2}^{\ell} W_{i}\right)\langle x \frown y \frown z\rangle \\
\Subset_{\bar{\gamma}} B\langle x \frown y \frown z\rangle
\end{array}
$$

and therefore $P_{1} \backslash H_{\ell}^{*}\left(x, W_{1}, W_{2}, \ldots, W_{\ell}\right)$ is a subset of

$$
\begin{array}{r}
\left\{y \in P_{1} \mid\left(\exists z \in P_{2}\right) \Psi^{-1}\left(E_{\emptyset} \cup \bigcup_{i=1}^{j-\ell}\left(E_{x^{*}(i)} \backslash E_{x_{1}^{*}(i)}\right) \cup \bigcup_{i=1}^{\ell} W_{i}\right)\langle x \frown y \frown z\rangle\right. \\
\left.\Subset_{\bar{\gamma}} B\langle x \frown y \frown z\rangle\right\}
\end{array}
$$

and Claim 4 now follows from Definition 3.1 and inequality (4.16).

Note that the second alternative of the hypothesis of Claim 3 includes the possibility that $j=\ell$ and $x=\emptyset$. Indeed, in the case that $j=\ell$ the hypothesis is automatically satisfied since $\sigma_{1}(\Theta(\emptyset))=\sigma_{1}\left(C_{j}\right)=\sigma_{1}(\emptyset)=0$. (Recall that the proof of Sublemma 4.1 began by eliminating the case $j=1$.) Hence, by an argument using inequalities (4.5) and (4.6) as in the first case, there is $\left(W_{1}, W_{2}, \ldots, W_{j}\right) \in \mathcal{P}(M)^{j}$ such that $\emptyset \in H_{j}\left(W_{1}, W_{2}, \ldots, W_{j}\right)$ and $|W|<(2 j+1) \bar{\xi} M$, where $W=E_{\emptyset} \cup \bigcup_{i=1}^{j} W_{i}$. From Claim 4 it follows that

$$
\Psi^{-1}\left(\bigcup_{i=1}^{j-j}\left(E_{x^{*}(i)} \backslash E_{x_{1}^{*}(i)}\right) \cup W\right)\langle\emptyset\rangle \Subset_{\bar{\gamma}} B\langle\emptyset\rangle
$$

and hence $\Psi^{-1} W \Subset_{\bar{\gamma}} B$. This establishes that Sublemma 4.1 holds.

With Sublemma 4.1 in place it is now possible to consider the general case. Given $B \subseteq\left(P_{1} \times P_{2}\right)^{j}$ let $B_{0}=\left\{z \in B\left|k_{1} \geq\right| E_{z_{1}^{*}(j)} \mid\right\}$ and let $B_{1}=B \backslash B_{0}$. It has already been shown that there is $W \subseteq M$ such that $|W|<(2 j+1) \bar{\xi} M$ and $(\Psi \uparrow$ $\left.B_{0}\right)^{-1} W \Subset_{\bar{\gamma}} B_{0}$. It will be shown by induction on $j$ that if $\left(\Psi \uparrow B_{0}\right)^{-1}(W) \Subset_{\bar{\gamma}} B_{0}$, then

$$
\mu\left(\left\{U \subseteq M \mid \Psi^{-1}(W \cup U) \Subset_{\gamma} B\right\}\right)>\beta(j),
$$

noticing the role $\gamma$ rather than $\bar{\gamma}$. Observe that the hypothesis includes the case that $B_{0}=\emptyset$.

If $j=1$, let $B_{k}^{*}=\left\{x \in P_{1} \mid \emptyset \neq B\langle x\rangle \subseteq B_{k}\right\}$ for $k<2$. Then by analyzing the meaning of Definition 3.1 for $\left(\Psi\left\lceil B_{0}\right)^{-1}(W) \Subset_{\bar{\gamma}} B_{0}\right.$ there are two cases to consider corresponding to conditions (3.1) and (3.2). The first is that there is some $x \in B_{0}^{*}$ such that $\sigma_{2}\left(\left\{y \in P_{2} \mid \Psi(x \frown y) \notin W\right\}\right)<\bar{\gamma}$. Then, since $B_{0}\langle x\rangle=B\langle x\rangle$, it is immediate that $\Psi^{-1}(W) \Subset_{\bar{\gamma}} B$ and hence $\Psi^{-1}(W \cup U) \Subset_{\gamma} B$ for every $U \subseteq M$. (Note that the assertion that $B_{0}\langle x\rangle=B\langle x\rangle$ is only true because $j=1$.) The second 
possibility is that $\sigma_{1}\left(\left\{x \in P_{1} \mid B_{0}\langle x\rangle \neq \emptyset\right.\right.$ and $\left.\left.\left(\forall y \in B_{0}\langle x\rangle\right) \Psi\left(x^{\frown} y\right) \notin W\right\}\right)<\bar{\gamma}$. In this case for each $x \in B_{1}^{*}$,

$$
\mu\left(\left\{U \subseteq M \mid U \cap E_{x}=\emptyset\right\}\right)=(1-\bar{\xi})^{\left|E_{x}\right|} \leq(1-\bar{\xi})^{k_{1}}<\bar{\delta}
$$

and so

$$
\int_{\mathcal{P}(M)} \sigma_{1}\left(\left\{x \in B_{1}^{*} \mid U \cap E_{x}=\emptyset\right\}\right) d \mu(U)=\int_{B_{1}^{*}} \mu\left(\left\{U \subseteq M \mid U \cap E_{x}=\emptyset\right\}\right) d \sigma_{1}(x)<\bar{\delta}
$$

and so

$$
\mu\left(\left\{U \subseteq M \mid \sigma_{1}\left(\left\{x \in B_{1}^{*} \mid U \cap E_{x}=\emptyset\right\}\right)<\bar{\gamma}\right\}\right)>1-\frac{\bar{\delta}}{\bar{\gamma}} \geq \beta(1) .
$$

This immediately implies that $\mu\left(\left\{U \subseteq M \mid \Psi^{-1}(U) \Subset_{\bar{\gamma}} B_{1}\right\}\right)>\beta(1)$ and hence

$$
\mu\left(\left\{U \subseteq M \mid \Psi^{-1}(U \cup W) \Subset_{\gamma} B\right\}\right)>\beta(1),
$$

as required.

If $j>1$ and $\left(\Psi \nmid B_{0}\right)^{-1} W \Subset_{\bar{\gamma}} B_{0}$, then according to Definition 3.1 there are two cases to consider corresponding to conditions (3.1) and (3.2). The first, corresponding to condition (3.1), is that there is some $x \in P_{1}$ such that $B_{0}\langle x\rangle \neq \emptyset$ and $\sigma_{2}\left(P_{2} \backslash G\right)<\bar{\gamma}$, where

$$
G=\left\{y \in P_{2} \mid\left(\Psi \uparrow B_{0}\right)^{-1}(W)\left\langle x^{\frown} y\right\rangle \Subset_{\bar{\gamma}} B_{0}\langle x \frown y\rangle \text { or } B_{0}\langle x \frown y\rangle=\emptyset\right\} .
$$

Observe that if $B_{0}\langle x \frown y\rangle=\emptyset$, then trivially $\left(\Psi \uparrow B_{0}\langle x \frown y\rangle\right)^{-1}(W) \Subset_{\bar{\gamma}} B_{0}\langle x \frown y\rangle$ and so, by the induction hypothesis, for each $y \in G$,

$$
\mu\left(\left\{U \subseteq M \mid \Psi^{-1}(W \cup U)\left\langle x^{\frown} y\right\rangle \Subset_{\gamma} B\left\langle x^{\frown} y\right\rangle\right\}\right) \geq \beta(j-1) .
$$

Integrating and applying Fubini's Theorem yields that

$$
\begin{gathered}
\int_{\mathcal{P}(M)} \sigma_{2}\left(\left\{y \in G \mid \Psi^{-1}(W \cup U)\langle x \frown y\rangle \notin_{\gamma} B\langle x \frown y\rangle\right\}\right) d \mu(U) \\
=\int_{G} \mu\left(\left\{U \subseteq M \mid \Psi^{-1}(W \cup U)\langle x \frown y\rangle \notin_{\gamma} B\langle x \frown y\rangle\right\}\right) d \sigma_{2}(y) \leq 1-\beta(j-1)
\end{gathered}
$$

and applying Markov's Inequality yields that

$$
\begin{aligned}
\mu\left(\left\{U \subseteq M \mid \sigma_{2}\left(\left\{y \in G \mid \Psi^{-1}(W \cup U)\langle x \frown y\rangle \notin_{\gamma} B\langle x \frown y\rangle\right\}\right)<\bar{\gamma}\right\}\right) \\
\quad>1-\frac{1-\beta(j-1)}{\bar{\gamma}}
\end{aligned}
$$

and the right-hand side of this inequality is at least $\beta(j)$. This suffices because the inequality $\sigma_{2}\left(P_{2} \backslash G\right)<\bar{\gamma}$ implies that

$$
\mu\left(\left\{U \subseteq M \mid \sigma_{2}\left(\left\{y \in P_{2} \mid \Psi^{-1}(W \cup U)\left\langle x^{\frown} y\right\rangle \notin_{\gamma} B\left\langle x^{\frown} y\right\rangle\right\}\right)<\gamma\right\}\right)>\beta(j)
$$

and hence $\mu\left(\left\{U \subseteq M \mid \Psi^{-1}(W \cup U) \Subset_{\gamma} B\right\}\right)>\beta(j)$.

The other possibility, corresponding to condition (3.2), is that if $G$ is defined to be

$$
\left\{x \in P_{1} \mid\left(\exists y \in P_{2}\right) \Psi^{-1}(W)\langle x \frown y\rangle \Subset_{\bar{\gamma}} B_{0}\left\langle x^{\frown} y\right\rangle \text { or } B_{0}\langle x\rangle=\emptyset\right\},
$$

then $\sigma_{1}\left(P_{1} \backslash G\right)<\bar{\gamma}$. Choose any function $y: G \rightarrow P_{2}$ such that if $B_{0}\langle x\rangle \neq \emptyset$, then $\Psi^{-1}(W)\langle x \frown y(x)\rangle \Subset_{\bar{\gamma}} B_{0}\langle x \frown y(x)\rangle$ and, if $B\langle x\rangle \neq \emptyset=B_{0}\langle x\rangle$, then $B\langle x \frown y(x)\rangle \neq \emptyset$. For each $x \in G$, applying the induction hypothesis to either $\Psi \backslash B_{0}\langle x \frown y(x)\rangle$ or $\Psi \uparrow B\langle x \frown y(x)\rangle$ yields that

$$
\mu\left(\left\{U \subseteq M \mid \Psi^{-1}(W \cup U)\langle x \frown y(x)\rangle \Subset_{\gamma} B\langle x \frown y(x)\rangle\right\}\right) \geq \beta(j-1) .
$$


Integrating over $x$ and applying Fubini's Theorem and Markov's Inequality as before yields that

$$
\begin{aligned}
\mu\left(\left\{U \subseteq M \mid \sigma_{1}\left(\left\{x \in G \mid \Psi^{-1}(W \cup U)\left\langle x^{\frown} y(x)\right\rangle \notin_{\gamma} B\left\langle x^{\frown} y(x)\right\rangle\right\}\right)<\bar{\gamma}\right\}\right) \\
\quad>1-\frac{1-\beta(j-1)}{\bar{\gamma}}
\end{aligned}
$$

and the argument can now be completed exactly as in the previous case.

Finally apply inequality (4.6) and Sublemma 4.1 to find $W$ and $U$ such that $\Psi^{-1}(W \cup U) \Subset_{\gamma} B$ and $|W|<(2 j+1) \bar{\xi} M$ and $|U|<2 \bar{\xi} M$. Hence $|W \cup U|<$ $2(j+1) \bar{\xi} M=\xi M$, as required.

Corollary 4.1. For any $n \in \mathbb{N}$ there is $M(n) \in \mathbb{N}$ such that $\nu\left(\mathcal{P}_{2^{-n}}(M(n))\right)>n$.

Proof. Let $M(n)=M\left(2^{-n}, \gamma, n\right)$ be as defined in Lemma 4.2 with $\gamma=$ $\min \left(\epsilon(j), K^{p} j^{p} J^{*}(j)^{p / q}\right) / 2$ and apply Lemma 4.2 and the version of Lemma 3.3 for $\left(\epsilon^{*}, L^{*}\right)$-fine probability spaces.

\section{Applichtions And Questions}

In order to apply Theorem 2.1 it is necessary to look for families satisfying the hypotheses of Definition 2.1 and inequality (2.2). Several such exist in the literature.

Theorem 5.1 (Stein 12]). If $\Lambda$ is as defined in Example 2.1 and if $k \geq 3$, then the maximal operator $M_{\Lambda}$ is bounded.

Theorem 5.2 (Bourgain [1, but see also Marstrand [8]). If $\Lambda$ is as defined in Example 2.1 and if $k=2$, then the maximal operator $M_{\Lambda}$ is bounded.

Theorem 5.3 (Falconer [3] and Marstrand [7]). If $\Lambda$ is as defined in Example 2.2, then the maximal operator $M_{\Lambda}$ is bounded.

Corollary 5.1. For $k \geq 2$ it is consistent that every set of reals of size $\aleph_{1}$ is null yet there are $\aleph_{1}$ spheres in $\mathbb{R}^{k}$ whose union is not null in $\mathbb{R}^{k}$.

Proof. Use Theorem 2.1 and Theorem 5.1 if $k \geq 3$ and Theorem 5.2 if $k=2$.

Corollary 5.2. It is consistent that every set of reals of size $\aleph_{1}$ is null yet there are $\aleph_{1}$ planes in the Euclidean 3-space whose union is not null.

Proof. Use Theorem 2.1 and Theorem 5.3 .

Since Corollaries 5.1 and 5.2 can both be obtained by appealing to Besicovitch duality arguments the key point here is that the theorems quoted actually apply to much broader classes of sets. For example, Theorem 5.2 actually yields that the maximal operator defined for the boundary of a smooth centrally symmetric convex body in $\mathbb{R}^{2}$ is bounded, and hence one gets as a corollary that for any such set $B$ it is consistent that every set of reals of size $\aleph_{1}$ is null yet there are $\aleph_{1}$ homothetic copies of $B$ whose union is not null.

It is curious that, while the analogue of Corollary 5.2 for lines in the plane in other words Komjáth's original question - remains true by the results of [11, Theorem 2.1 cannot be applied because the associated maximal operator is not bounded. The non-boundedness follows from the existence of a Besicovitch set, a null set in the plane containing line segments in all directions (see $\S 7.1$ of [4]). In this context it is worth mentioning a result of Talagrand [13, which shows the existence 
of an analogue of the Besicovitch set for circles. In particular, he shows that there exists a family of circles of the plane whose union is of measure zero, but such that the set of the centres is of non-zero linear measure. This provides the same sort of obstacle as the Besicovitch set to proving the consistency of: Every set of reals of size $\aleph_{1}$ is null yet there are $\aleph_{1}$ circles in the plane with centres on the $x$-axis whose union is not null. One might hope to obtain this by applying the duality betweeen lines and circles via complex inversion to the answer to Komjáth's question. However, this would require that in the model answering Komjáth's question the set of lines of positive measure would have to be of a restricted type in order that the circles obtained by complex inversion all had their centres on a straight line. This remains an open question with the possibility that Talagrand's construction can be used to provide a positive result.

The introduction remarked that a broad class of problems concerns determining which homemorphic images $W$ of the unit interval in $\mathbb{R}^{n}$ have the property that $\aleph_{1}$ isometric copies of $W$ can have non-null union while $\aleph_{1}$ sized sets of reals are all null. Even very natural examples of $W$ provide open problems.

Question 5.1. Is it consistent that every set of reals of size $\aleph_{1}$ is null yet there are $\aleph_{1}$ spirals in $\mathbb{R}^{3}$ whose union is not null?

This is actually a family of questions depending on precisely what is meant by a spiral. If

$$
\Lambda(t)=\{(t \cos (x), t \sin (x), t x) \mid 0 \leq x \leq 1\}
$$

for $t \in[1,2]$, then Pramanik and Seeger have shown [9] that the associated maximal function is bounded. However, condition (3) of Definition 2.1 does not hold in this case. On the other hand, if

$$
\Lambda(t, s)=\{(t \cos (x), t \sin (x), s x) \mid 0 \leq x \leq 1\}
$$

for $(t, s) \in[1,2]^{2}$, then it can be shown that condition (3) of Definition 2.1 does hold, but the associated maximal function is not known to be bounded. However, 9] does have results in this direction.

Finally, there is the question of establishing consistent inequalities such as $\operatorname{add}\left(\mathcal{P}^{3}\right) \neq \operatorname{add}\left(\mathcal{C}^{2}\right)$, in other words inequalities involving cardinal invariants for both of which the associated maximal operator is bounded. Several difficulties would have to be overcome to make progress in this direction. First, substantial changes to the forcing partial order would be required since the objective now would be to cover, for example, ground model circles with a null set while keeping the measure of the ground model lines positive. Assuming this could be done, a more serious and potentially more interesting challenge, a challenge to harmonic analysis, would arise in finding the correct boundedness theorems for maximal operators.

\section{REFERENCES}

[1] J. Bourgain. Averages in the plane over convex curves and maximal operators. J. Analyse Math., 47:69-85, 1986. MR874045 (88f:42036)

[2] R. O. Davies. On accessibility of plane sets and differentiation of functions of two real variables. Proc. Cambridge Philos. Soc., 48:215-232, 1952. MR0045795(13:635d)

[3] K. J. Falconer. Continuity properties of $k$-plane integrals and Besicovitch sets. Math. Proc. Cambridge Philos. Soc., 87(2):221-226, 1980. MR553579 (81c:53067)

[4] K. J. Falconer. The geometry of fractal sets, volume 85 of Cambridge Tracts in Mathematics. Cambridge University Press, Cambridge, 1986. MR.867284 (88d:28001) 
[5] Kenneth Falconer. Fractal geometry. John Wiley \& Sons Ltd., Chichester, 1990. Mathematical foundations and applications. MR 1102677 (92j:28008)

[6] Peter Komjáth. Talk at summer meeting of the asl. Veszprém, Hungary, August 1992.

[7] J. M. Marstrand. Packing planes in $\mathbf{R}^{3}$. Mathematika, 26(2):180-183 (1980), 1979. MR575639 (81f:28007)

[8] J. M. Marstrand. Packing circles in the plane. Proc. London Math. Soc. (3), 55(1):37-58, 1987. MR887283 (88i:28012)

[9] Malabika Pramanik and Andreas Seeger. $L^{p}$ regularity of averages over curves and bounds for associated maximal operators. Amer. J. Math., 129(1):61-103, 2007. MR.2288738 $(2007 \mathrm{k}: 42034)$

[10] Andrzej Rosłanowski and Saharon Shelah. Norms on possibilities. I. Forcing with trees and creatures. Mem. Amer. Math. Soc., 141(671):xii+167 pp., 1999. MR.1613600 (2000c:03036)

[11] Saharon Shelah and Juris Steprāns. Comparing the uniformity invariants of null sets for different measures. Adv. Math., 192(2):403-426, 2005. MR2128705 (2006h:03044)

[12] Elias M. Stein. Maximal functions. I. Spherical means. Proc. Nat. Acad. Sci. U.S.A., 73(7):2174-2175, 1976. MR0420116 (54:8133a)

[13] Michel Talagrand. Sur la mesure de la projection d'un compact et certaines familles de cercles. Bull. Sci. Math. (2), 104(3):225-231, 1980. MR592470(82b:28004)

Department of Mathematics, York University, 4700 Keele Street, Toronto, Ontario, CANADA M3J 1P3

E-mail address: steprans@yorku.ca 